\title{
GAUSSIAN AND NON-GAUSSIAN PROCESSES OF ZERO POWER VARIATION
}

\author{
Francesco Russo ${ }^{1,2}$ AND Frederi Viens ${ }^{3}$
}

\begin{abstract}
We consider a class of stochastic processes $X$ defined by $X(t)=\int_{0}^{T} G(t, s) \mathrm{d} M(s)$ for $t \in[0, T]$, where $M$ is a square-integrable continuous martingale and $G$ is a deterministic kernel. Let $m$ be an odd integer. Under the assumption that the quadratic variation $[M]$ of $M$ is differentiable with $\mathbf{E}\left[|\mathrm{d}[M](t) / \mathrm{d} t|^{m}\right]$ finite, it is shown that the $m$ th power variation

$$
\lim _{\varepsilon \rightarrow 0} \varepsilon^{-1} \int_{0}^{T} \mathrm{~d} s(X(s+\varepsilon)-X(s))^{m}
$$

exists and is zero when a quantity $\delta^{2}(r)$ related to the variance of an increment of $M$ over a small interval of length $r$ satisfies $\delta(r)=o\left(r^{1 /(2 m)}\right)$. When $M$ is the Wiener process, $X$ is Gaussian; the class then includes fractional Brownian motion and other Gaussian processes with or without stationary increments. When $X$ is Gaussian and has stationary increments, $\delta$ is $X$ 's univariate canonical metric, and the condition on $\delta$ is proved to be necessary. In the non-stationary Gaussian case, when $m=3$, the symmetric (generalized Stratonovich) integral is defined, proved to exist, and its Itô's formula is established for all functions of class $C^{6}$.
\end{abstract}

Mathematics Subject Classification. 60G07, 60G15, 60G48, 60H05.

Received August 9, 2012. Revised July 21, 2014.

\section{INTRODUCTION}

The purpose of this article is to study wide classes of processes with zero cubic variation, and more generally, zero variation of any odd order. Before summarizing our results, we give a brief historical description of the topic of $p$-variations, as a basis for our motivations.

\subsection{Historical background}

The $p$-variation of a function $f:[0, T] \rightarrow \mathbf{R}$ is the supremum over all the possible partitions $\left\{0=t_{0}<\ldots<\right.$ $\left.t_{N}=T\right\}$ of $[0, T]$ of the quantity $\sum_{i=0}^{N-1}\left|f\left(t_{i+1}\right)-f\left(t_{i}\right)\right|^{p}$. The analytic monograph [9] contains an interesting study on this concept, showing that a $p$-variation function is the composition of an increasing function and

\footnotetext{
Keywords and phrases. Power variation, martingale, calculus via regularization, Gaussian processes, generalized Stratonovich integral, non-Gaussian processes.

1 ENSTA-ParisTech. Unité de Mathématiques appliquées, 828, bd des Maréchaux, 91120 Palaiseau, France.

2 INRIA Rocquencourt, Projet MathFi and Cermics, École des Ponts, Rocquencourt, France.

3 Department of Statistics, Purdue University, 150 N. University St., West Lafayette, IN 47907-2067, USA.

viens@stat . purdue .edu
} 
a Hölder-continuous function. The analytic notion of $p$-variation precedes stochastic calculus and processes (see [9]).

It was rediscovered in stochastic analysis in the context of pathwise stochastic calculus, starting with $p=2$ as in the fundamental paper [16] of H. Föllmer. Dealings with $p$-variations and their stochastic applications, particularly to rough path and other recent integration techniques for fractional Brownian motion (fBm) and related processes, are described at length for instance in the books $[12,17,24]$, which also contain excellent bibliographies on the subject. Prior to this, power variations could be seen as related to oscillations of processes in [4], and some specific cases had been treated, such as local time processes (see [32]).

The Itô stochastic calculus for semimartingales defines a quadratic variation $[S]$ of a semimartingale $S$, instead of its 2-variation, as the limit in probability of $\sum_{i=0}^{N-1}\left|S\left(t_{i+1}\right)-S\left(t_{i}\right)\right|^{2}$ over the smaller set of partitions whose mesh tends to 0 , instead of the pathwise supremum over all partitions, in the hopes of making it more likely to have a finite limit. This is indeed the case for standard Brownian motion $M=B$, where its 2-variation $[B]$ is a.s. infinite, but its quadratic variation is equal to $T$. To reconcile 2 -variations with the finiteness of $[B]$, many authors have proposed restricting the supremum over dyadic partitions. But there is a fundamental difference between the deterministic and stochastic versions of "variation", since in Itô calculus, quadratic variation is associated with the notion of covariation (also known as joint quadratic variation), something which is not present in analytic treatments of 2-variation. The co-variation $\left[S^{1}, S^{2}\right]$ of two semimartingales $S^{1}, S^{2}$ is obtained by polarization, using again a limit in probability when the partition mesh goes to zero.

To work with a general class of processes, the tools of Itô calculus would nonetheless restrict the study of covariation to semimartingales. In [34], the authors enlarged the notion of covariation to general processes, in an effort to create a more efficient stochastic calculus tool to go beyond semimartingales, by considering regularizations instead of discretizations. Drawing some inspiration from the classical fact that a continuous $f:[0, T] \rightarrow \mathbf{R}$ has finite variation (1-variation) if and only if $\lim _{\varepsilon \rightarrow 0} \frac{1}{\varepsilon} \int_{0}^{T}|f(s+\varepsilon)-f(s)| \mathrm{d} s$ exists, for two processes $X$ and $Y$, their covariation $[X, Y](t)$ is the limit in probability, when $\varepsilon$ goes to zero, of

$$
[X, Y]_{\varepsilon}(t)=\varepsilon^{-1} \int_{0}^{t}(X(s+\varepsilon)-X(s))(Y(s+\varepsilon)-Y(s)) \mathrm{d} s ; \quad t \geq 0 .
$$

$[X, Y]$ coincides with the classical covariation for continuous semimartingales. The processes $X$ such that $[X, X]$ exists are called finite quadratic variation processes, and were analyzed in $[15,35]$.

The notion of covariation was extended in [14] to more than two processes: the $n$-covariation $\left[X^{1}, X^{2}, \ldots, X^{n}\right]$ of $n$ processes $X^{1}, \ldots, X^{n}$ is as in formula (1.1), but with a product of $n$ increments, with specific analyses for $n=4$ for fBm with "Hurst" parameter $H=1 / 4$ in [19]. If $X=X^{1}=X^{2}=X^{3}$ we denote $[X ; 3]:=[X, X, X]$, which is called the cubic variation, and is one of the main topics of investigation in our article. This variation is the limit in probability of

$$
[X, 3]_{\varepsilon}(t):=\varepsilon^{-1} \int_{0}^{t}(X(s+\varepsilon)-X(s))^{3} \mathrm{~d} s,
$$

when $\varepsilon \rightarrow 0$. (1.2) involves the signed cubes $(X(s+\varepsilon)-X(s))^{3}$, which has the same sign as the increment $X(s+\varepsilon)-X(s)$, unlike the case of quadratic or 2-variation, or of the so-called strong cubic variation, where absolute values are used inside the cube function. Consider the case where $X$ is a $\mathrm{fBm} B^{H}$ with Hurst parameter $H \in(0,1)$. For $\mathrm{fBm},[20]$ establish that $[X, 3] \equiv 0$ if $H>1 / 6$ and $[X, 3]$ does not exist if $H<1 / 6$, while for $H=1 / 6$, the regularization approximation $[X, 3]_{\varepsilon}(t)$ converges in law to a normal law for every $t>0$. This phenomenon was confirmed for the related finite-difference approximating sequence of $[X, 3](t)$ which also converges in law to a Gaussian variable; this was proved in ([31], Thm. 10) by using the the so-called Breuer-Major central limit theorem for stationary Gaussian sequences [8].

A practical significance of the cubic variation lies in its well-known ability to guarantee the existence of (generalized symmetric) Stratonovich integrals, and their associated Itô-Stratonovich formula, for various highly irregular processes. This was established in [20] in significant generality; technical conditions therein were proved to apply to fBm with $H>1 / 6$, and can extend to similar Gaussian cases with canonical metrics that are bounded above and below by multiples of the fBm's, for instance the bi-fractional Brownian motion treated in [33]. A variant on [20]'s Itô formula was established previously in [14] for less irregular processes: if $X$ (not necessarily 
Gaussian) has a finite strong cubic variation, so that $[X, 3]$ exists (but may not be zero), for $f \in C^{3}(\mathbf{R})$, $f\left(X_{t}\right)=f\left(X_{0}\right)+\int_{0}^{t} f^{\prime}\left(X_{s}\right) d^{\circ} X-\frac{1}{12} \int_{0}^{t} f^{\prime \prime \prime}\left(X_{s}\right) d[X, 3](s)$, which involves the symmetric-Stratonovich integral of [36], and a Lebesgue-Stieltjes integral. In [29], an analogous formula is obtained for fBm with $H=1 / 6$, but in the sense of distribution laws only: $\int_{0}^{t} f^{\prime}\left(X_{s}\right) d^{\circ} X$ exist only in law, and $\int_{0}^{t} f^{\prime \prime \prime}\left(X_{s}\right) d[X, 3](s)$ is replaced by a conditionally Wiener integral defined in law by replacing $[X, 3]$ with a term $\kappa W$, where $W$ is the independent Wiener process identified in [31].

\subsection{Specific motivations}

Our work herein is motivated by the properties described in the previous paragraph, particularly as in [20]. We want to avoid situations where Itô formulas can only be established in law, i.e. involving conditionally Wiener integrals defined as limits in a weak sense. Thus we study scales where this term vanishes in a strong sense, while staying as close to the threshold $H=1 / 6$ as possible. Other types of stochastic integrals for fBm and related irregular Gaussian processes make use of the Skorohod integral, identified as a divergence operator on Wiener space (see [30] and also $[2,6,11,22,25]$ ), and rough path theory (see [17,24]). The former method is not restrictive in how small $H$ can be (see [25]), but is known not to represent a pathwise notion of integral; the latter is based on a true pathwise strategy and requires giving a Lévy-type area or iterated integrals a priori. In principal the objective of the rough path approach is not to link any discretization (or other approximation) scheme. These provide additional motivations for studying the regularlization methodology of [34] or [36], which does not feature these drawbacks for $H>1 / 6$.

We come back to the cubic variation approximation $[X, 3]$ defined via the limit of (1.2). The reasons for which $[X, 3]=0$ for fBm with $H>1 / 6$, which is considerably less regular than the threshold $H>1 / 3$ one has for $H$-Hölder-continuous deterministic functions, are the odd symmetry of the cube function, and the accompanying probabilistic symmetries of the process $X$ itself (e.g. Gaussian property). This doubling improvement over the deterministic case does not typically hold for non-symmetric variations: $H$ needs to be larger to guarantee existence of the variation; for instance, when $X$ is $\mathrm{fBm}$, its strong cubic variation, defined as the limit in probability of $\varepsilon^{-1} \int_{0}^{t}|X(s+\varepsilon)-X(s)|^{3} \mathrm{~d} s$, exists for $H \geq 1 / 3$ only.

Finally, some brief notes in the case where $X$ is fBm with $H=1 / 6$. This threshold is a critical value since, as mentioned above, whether in the sense of regularization or of finite-difference, the approximating sequences of $[X, 3](t)$ converge in law to Gaussian laws. In contrast to these normal convergences, in our article, we show as a preliminary result (Prop. 3.2 herein), that $[X, 3]_{\varepsilon}$ does not converge in probability for $H=1 / 6$; the non-convergence of $[X, 3]_{\varepsilon}$ in probability for $H<1 / 6$ was known previously.

\subsection{Summary of results and structure of article}

This article investigates the properties of cubic and other odd variations for processes which may not be self-similar, or have stationary increments, or be Gaussian, when they have $\alpha$-Hölder-continuous paths; this helps answer to what extent the threshold $\alpha>1 / 6$ is sharp for $[X, 3]=0$. We consider processes $X$ defined on $[0, T]$ by a Volterra representation

$$
X(t)=\int_{0}^{T} G(t, s) \mathrm{d} M(s),
$$

where $M$ is a square-integrable martingale on $[0, T]$, and $G$ is a non-random measurable function on $[0, T]^{2}$, which is square-integrable in $s$ with respect to $d[M]_{s}$ for every fixed $t$. The quadratic variations of these martingalebased convolutions was studied in [13]. The "Gaussian" case results when $M$ is the standard Wiener process (Brownian motion) $W$.

In this paper, we concentrate on processes $X$ which are not more regular than standard Brownian motion; this irregularity is expressed via a concavity condition on the squared canonical metric $\delta^{2}(s, t)=$ $\mathbf{E}\left[\left(X(t)-X(s)^{2}\right)\right]$. It is not a restriction since the main interest of our results occurs around the Hölder 
exponent $1 /(2 m)$ for odd $m \geq 3$, and processes which are more regular than Brownian motion can be treated using classical non-probabilistic tools such as the Young integral.

After providing some definitions (Sect. 2), our first main finding is that the processes with zero odd $m$ th variation (same definition as for $[X, 3]=0$ in (1.2) but with $m$ replacing 3) are those which are better than $1 /(2 m)$-Hölder-continuous in the $L^{2}(\Omega)$-sense, whether for Gaussian processes (Sect. 3), or non-Gaussian ones (Sect. 4). Specifically,

- for $X$ Gaussian with stationary increments $(i . e . \delta(s, t)=\delta(t-s)$ ), for any odd integer $m \geq 3,[X, m]=0$ if and only if $\delta(r)=o\left(r^{1 /(2 m)}\right)$ for $r$ near 0 (Thm. 3.6);

- for $X$ Gaussian but not necessarily with stationary increments, for any odd integer $m \geq 3,[X, m]=0$ if $\delta^{2}(s, s+r)=o\left(r^{1 /(2 m)}\right)$ for $r$ near 0 uniformly in $s$. (see Thm. 3.8; this holds under a technical nonexplosion condition on the mixed partial derivative of $\delta^{2}$ near the diagonal; see Examples 3.9 and 3.10 for a wide class of Volterra-convolution-type Gaussian processes with non-stationary increments which satisfy the condition).

- for $X$ non-Gaussian based on a martingale $M$, for any odd integer $m \geq 3$, with $\Gamma(t):=$ $\left(\mathbf{E}\left[(d[M] / \mathrm{d} t)^{m}\right]\right)^{1 /(2 m)}$ if it exists, we let $Z(t):=\int_{0}^{T} \Gamma(s) G(t, s) \mathrm{d} W(s)$. This $Z$ is a Gaussian process; if it satisfies the conditions of Theorem 3.6 or Theorem 3.8, then $[X, m]=0$. (Thm. 4.1 on Sect. 4; Prop. 4.2 provides examples of wide classes of martingales and kernels for which the assumptions of Thm. 4.1 are satisfied, with details on how to construct examples and study their regularity properties.)

Our results shows how broad a class of processes, based on martingale convolutions with only $m$ moments, one can construct which have zero odd $m$ th variation, under conditions which are the same in terms of regularity as in the case of Gaussian processes with stationary increments, where we prove sharpness. Note that $X$ itself can be far from having the martingale property, just as it is generally far from standard Brownian motion in the Gaussian case. Our second main result is an application to weighted variations, generalized Stratonovich integration, and an Itô formula (Sect. 5).

- Under the conditions of Theorem 3.8 (general Gaussian case), and an additional coercivity condition, for every bounded measurable function $g$ on $\mathbf{R}$ (see Thm. 5.1),

$$
\lim _{\varepsilon \rightarrow 0} \frac{1}{\varepsilon^{2}} \mathbf{E}\left[\left(\int_{0}^{T} \mathrm{~d} u\left(X_{u+\varepsilon}-X_{u}\right)^{m} g\left(\frac{X_{u+\varepsilon}+X_{u}}{2}\right)\right)^{2}\right]=0 .
$$

If $m=3$, by results in [20], Theorem 5.1 implies that for any $f \in C^{6}(\mathbf{R})$ and $t \in[0, T]$, the Itô's formula $f\left(X_{t}\right)=f\left(X_{0}\right)+\int_{0}^{t} f^{\prime}\left(X_{u}\right) d^{\circ} X_{u}$ holds, where the integral is in the symmetric (generalized Stratonovich) sense. (Thm. 5.1 and its Cor. 5.2).

Some of the proofs of our theorems are relegated to the Appendix.

\subsection{Relation with other recent work}

The authors of the paper [21] consider, as we do, stochastic processes which can be written as Volterra integrals with respect to martingales. Their "fractional martingale", which generalizes Riemann-Liouville fBm, is a special case of the processes we consider in Section 4, with $K(t, s)=(t-s)^{H-1 / 2}$. The authors' motivation is to prove an analogue of the famous Lévy characterization of Brownian motion as the only continuous square-integrable martingale with a quadratic variation equal to $t$. They provide similar necessary and sufficient conditions based on the $1 / H$-variation for a process to be $\mathrm{fBm}$. This is a different aspect of the theory than our motivation to study necessary and sufficient conditions for a process to have vanishing (odd) cubic variation, and its relation to stochastic calculus. The value $H=1 / 6$ is mentioned in the context of the stochastic heat equation driven 
by space-time white-noise, in which discrete trapezoidal sums converge in distribution (not in probability) to a conditionally independent Brownian motion (see [10,31]).

To find a similar motivation to ours, one may look at the recent result of [28], where the authors study the central and non-central behavior of weighted Hermite variations for $\mathrm{fBm}$. Using the Hermite polynomial of order $m$ rather than the power- $m$ function, they show that the threshold value $H=1 /(2 m)$ poses an interesting open problem, since above this threshold (but below $H=1-1 /(2 m)$ ) one obtains Gaussian limits (these limits are conditionally Gaussian when weights are present, and can be represented as Wiener integrals with respect to an independent Brownian motion), while below the threshold, degeneracy occurs. The behavior at the threshold was worked out for $H=1 / 4, m=2$ in [28], boasting an exotic correction term with an independent Brownian motion, while the general open problem of Hermite variations with $H=1 /(2 m)$ was settled in [27]. More questions arise, for instance, with a similar result in [26] for $H=1 / 4$, but this time with bidimensional fBm, in which two independent Brownian motions are needed to characterize the exotic correction term. Compared to the above works, our work situates itself by

- establishing necessary and sufficient conditions for nullity of the odd $m$ th variation, around the threshold regularity value $H=1 /(2 m)$, for general Gaussian processes with stationary increments, showing in particular that self-similarity is not related to this nullity, and that the result works for all odd integers, thanks only to the problem's symmetries;

- showing that our method is able to consider processes that are far from Gaussian and still yield sharp sufficient conditions for nullity of odd variations, since our base noise may be a generic martingale with only a few moments.

Our ability to prove an Itô formula for processes which are far from self-similar or from having stationary increments attests to our method's strength.

\section{Definitions}

We recall our process $X$ defined for all $t \in[0, T]$ by (1.3). For any odd integer $m \geq 3$, let the odd $\varepsilon$ - $m$ th variation of $X$ be defined by

$$
[X, m]_{\varepsilon}(T):=\frac{1}{\varepsilon} \int_{0}^{T} \mathrm{~d} s(X(s+\varepsilon)-X(s))^{m} .
$$

The odd variation is different from the absolute (or strong) variation where the power function $x^{m}$ is replaced by

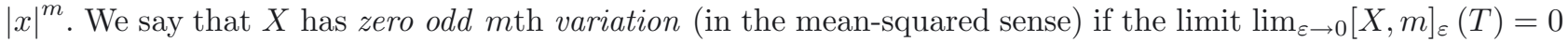
holds in $L^{2}(\Omega)$.

The canonical metric $\delta$ of a stochastic process $X$ is defined as the pseudo-metric on $[0, T]^{2}$ given by

$\delta^{2}(s, t):=\mathbf{E}\left[(X(t)-X(s))^{2}\right]$. The covariance function of $X$ is defined by $Q(s, t):=\mathbf{E}[X(t) X(s)]$. The special case of a centered Gaussian process is of primary importance; then the process's entire distribution is characterized by $Q$, or alternately by $\delta$ and the variances $\operatorname{var}(X(t))=Q(t, t)$, since we have $Q(s, t)=\frac{1}{2}\left(Q(s, s)+Q(t, t)-\delta^{2}(s, t)\right)$. We say that $\delta$ has stationary increments if there exists a function on $[0, T]$ which we also denote by $\delta$ such that $\delta(s, t)=\delta(|t-s|)$. Below, we will refer to this situation as the stationary case. This is in contrast to usual usage of this appellation, which is stronger, since for example in the Gaussian case, it refers to the fact that $Q(s, t)$ depends only on the difference $s-t$; this would not apply to, say, standard or $\mathrm{fBm}$, while our definition does. In non-Gaussian settings, the usual way to interpret the "stationary" property is to require that the processes $X(t+\cdot)$ and $X(\cdot)$ have the same law, which is typically much more restrictive than our definition.

The goal of the next two sections is to define various general conditions under which a characterization of $\lim _{\varepsilon \rightarrow 0}[X, m]_{\varepsilon}(T)=0$ can be established. In particular, we aim to show that $X$ has zero odd $m$ th variation for 
well-behaved $M$ 's and $G$ 's if - and in some cases only if -

$$
\delta(s, t)=o\left(|t-s|^{1 /(2 m)}\right) .
$$

\section{Gaussian CASE}

We assume that $X$ is centered Gaussian. Then we can write $X$ as in formula (1.3) with $M=W$ a standard Brownian motion. The following elementary result is proved in the Appendix.

Lemma 3.1. If $m$ is an odd integer $\geq 3$, we have $\mathbf{E}\left[\left([X, m]_{\varepsilon}(T)\right)^{2}\right]=\sum_{j=0}^{(m-1) / 2} J_{j}$ where

$$
J_{j}:=\frac{1}{\varepsilon^{2}} \sum_{j=0}^{(m-1) / 2} c_{j} \int_{0}^{T} \int_{0}^{t} \mathrm{~d} t \mathrm{~d} s \Theta^{\varepsilon}(s, t)^{m-2 j} \operatorname{Var}[X(t+\varepsilon)-X(t)]^{j} \operatorname{Var}[X(s+\varepsilon)-X(s)]^{j},
$$

the $c_{j}$ 's are positive constants depending only on $j$, and

$$
\Theta^{\varepsilon}(s, t):=\mathbf{E}[(X(t+\varepsilon)-X(t))(X(s+\varepsilon)-X(s))] .
$$

Using $Q$ and $\delta, \Theta^{\varepsilon}(s, t)$ computes as the opposite of the planar increment of the canonical metric over the rectangle defined by its corners $(s, t)$ and $(s+\varepsilon, t+\varepsilon)$ :

$$
\Theta^{\varepsilon}(s, t)=\frac{1}{2}\left[-\delta^{2}(t+\varepsilon, s+\varepsilon)+\delta^{2}(t, s+\varepsilon)+\delta^{2}(s, t+\varepsilon)-\delta^{2}(s, t)\right]=:-\frac{1}{2} \Delta_{(s, t) ;(s+\varepsilon, t+\varepsilon)} \delta^{2} .
$$

\subsection{The case of critical fBm}

Before finding sufficient and possibly necessary conditions for various Gaussian processes to have zero cubic (or odd $m$ th) variation, we discuss the threshold case for the cubic variation of fBm. Recall that when $X$ is fBm with parameter $H=1 / 6$, as mentioned in the Introduction, it is known from ([20], Thm. 4.1 part (2)) that $[X, 3]_{\varepsilon}(T)$ converges in distribution to a non-degenerate normal law. However, there does not seem to be any place in the literature specifying whether the convergence may be any stronger than in distribution. We address this issue here.

Proposition 3.2. Let $X$ be an $f B m$ with Hurst parameter $H=1 / 6$. Then $X$ does not have a cubic variation (in the mean-square sense), by which we mean that $[X, 3]_{\varepsilon}(T)$ has no limit in $L^{2}(\Omega)$ as $\varepsilon \rightarrow 0$. In fact more is true: $[X, 3]_{\varepsilon}(T)$ has no limit in probability as $\varepsilon \rightarrow 0$.

To prove the proposition, we study the Wiener chaos representation and moments of $[X, 3]_{\varepsilon}(T)$ when $X$ is $\mathrm{fBm} ; X$ is given by (1.3) where $W$ is Brownian motion and the kernel $G$ is well-known (see Chaps. 1 and 5 in [30]). The next three lemmas are proved in the Appendix.

Lemma 3.3. Fix $\varepsilon>0$. Let $\Delta G_{s}(u):=G(s+\varepsilon, u)-G(s, u)$. Then $[X, 3]_{\varepsilon}(T)=\mathcal{I}_{1}+\mathcal{I}_{3}$ where

$$
\begin{aligned}
& \mathcal{I}_{1}:=\frac{3}{\varepsilon} \int_{0}^{T} \mathrm{~d} s \int_{0}^{T} \Delta G_{s}(u) \mathrm{d} W(u)\left(\int_{0}^{T}\left|\Delta G_{s}(v)\right|^{2} \mathrm{~d} v\right), \\
& \mathcal{I}_{3}:=\frac{6}{\varepsilon} \int_{0}^{T} \mathrm{~d} W\left(s_{3}\right) \int_{0}^{s_{3}} \mathrm{~d} W\left(s_{2}\right) \int_{0}^{s_{2}} \mathrm{~d} W\left(s_{1}\right) \int_{0}^{T}\left[\prod_{k=1}^{3} \Delta G_{s}\left(s_{k}\right)\right] \mathrm{d} s .
\end{aligned}
$$

The above lemma indicates the Wiener chaos decomposition of $[X, 3]_{\varepsilon}(T)$ into the term $\mathcal{I}_{1}$ of line $(3.2)$ which is in the first Wiener chaos (i.e. a Gaussian term), and the term $\mathcal{I}_{3}$ of line (3.3), in the third Wiener chaos. The next two lemmas contain information on the behavior of each of these two terms, as needed to prove Proposition 3.2. 
Lemma 3.4. $\mathcal{I}_{1}$ converges to 0 in $L^{2}(\Omega)$ as $\varepsilon \rightarrow 0$.

Lemma 3.5. $\mathcal{I}_{3}$ is bounded in $L^{2}(\Omega)$ for all $\varepsilon>0$, and does not converge in $L^{2}(\Omega)$ as $\varepsilon \rightarrow 0$.

Proof of Proposition 3.2. We prove the proposition by contradiction. Assume $[X, 3]_{\varepsilon}(T)$ converges in prob-

ability. For any $p>2$, there exists $c_{p}$ depending only on $p$ such that $\mathbf{E}\left[\left|\mathcal{I}_{1}\right|^{p}\right] \leq c_{p}\left(\mathbf{E}\left[\left|\mathcal{I}_{1}\right|^{2}\right]\right)^{p / 2}$ and $\mathbf{E}\left[\left|\mathcal{I}_{3}\right|^{p}\right] \leq c_{p}\left(\mathbf{E}\left[\left|\mathcal{I}_{3}\right|^{2}\right]\right)^{p / 2}$; this is a general fact about random variables in fixed Wiener chaos, and can be proved directly using Lemma 3.3 and the Burkhölder-Davis-Gundy's inequalities. Also see [7]. Therefore, since we have $\sup _{\varepsilon>0}\left(\mathbf{E}\left[\left|\mathcal{I}_{1}\right|^{2}\right]+\mathbf{E}\left[\left|\mathcal{I}_{3}\right|^{2}\right]\right)<\infty$ by Lemmas 3.4 and 3.5, we also get $\sup _{\varepsilon>0}\left(\mathbf{E}\left[\left|\mathcal{I}_{1}+\mathcal{I}_{3}\right|^{p}\right]\right)<\infty$ for any $p$. Therefore, by uniform integrability, $[X, 3]_{\varepsilon}(T)=\mathcal{I}_{1}+\mathcal{I}_{3}$ converges in $L^{2}(\Omega)$. In $L^{2}(\Omega)$, the terms $\mathcal{I}_{1}$ and $\mathcal{I}_{3}$ are orthogonal. Therefore, $\mathcal{I}_{1}$ and $\mathcal{I}_{3}$ must converge in $L^{2}(\Omega)$ separately. This contradicts the nonconvergence of $\mathcal{I}_{3}$ in $L^{2}(\Omega)$ obtained in Lemma 3.5. Thus $[X, 3]_{\varepsilon}(T)$ does not converge in probability.

\subsection{The case of stationary increments}

We prove a necessary and sufficient condition for having a zero odd $m$ th variation for Gaussian processes with stationary increments.

Theorem 3.6. Let $m \geq 3$ be an odd integer. Let $X$ be a centered Gaussian process on $[0, T]$ with stationary increments; its canonical metric is

$$
\delta^{2}(s, t):=\mathbf{E}\left[(X(t)-X(s))^{2}\right]=\delta^{2}(|t-s|)
$$

where the univariate function $\delta^{2}$ is assumed to be increasing and concave on $[0, T]$. Then $X$ has zero odd mth variation if and only if $\delta(r)=o\left(r^{1 /(2 m)}\right)$.

Proof.

Step 0. Setup. The derivative $\mathrm{d} \delta^{2}$ of $\delta^{2}$, in the sense of measures, is positive and bounded on $[0, T]$. By stationarity, $\operatorname{Var}[X(t+\varepsilon)-X(t)]=\delta^{2}(\varepsilon)$. Using the notation in Lemma 3.1, we get

$$
J_{j}=\varepsilon^{-2} \delta^{4 j}(\varepsilon) c_{j} \int_{0}^{T} \mathrm{~d} t \int_{0}^{t} \mathrm{~d} s \Theta^{\varepsilon}(s, t)^{m-2 j} .
$$

Step 1. Diagonal. We define the $\varepsilon$-diagonal $D_{\varepsilon}:=\{0 \leq t-\varepsilon<s<t \leq T\}$. Trivially using the Cauchy-Schwarz's inequality,

$$
\left|\Theta^{\varepsilon}(s, t)\right| \leq \sqrt{\operatorname{Var}[X(t+\varepsilon)-X(t)] \operatorname{Var}[X(s+\varepsilon)-X(s)]}=\delta^{2}(\varepsilon) .
$$

Hence, according to Lemma 3.1, the diagonal portion $\sum_{j=0}^{(m-1) / 2} J_{j, D_{\varepsilon}}$ of $\mathbf{E}\left[\left([X, m]_{\varepsilon}(T)\right)^{2}\right]$ can be bounded above, in absolute value, as:

$$
\begin{aligned}
\left|\sum_{j=0}^{(m-1) / 2} J_{j, D_{\varepsilon}}\right| & :=\left|\sum_{j=0}^{(m-1) / 2} \varepsilon^{-2} \delta^{4 j}(\varepsilon) c_{j} \int_{\varepsilon}^{T} \mathrm{~d} t \int_{t-\varepsilon}^{t} \mathrm{~d} s \Theta^{\varepsilon}(s, t)^{m-2 j}\right| \\
& \leq \frac{1}{\varepsilon^{2}} \sum_{j=0}^{(m-1) / 2} c_{j} \int_{\varepsilon}^{T} \mathrm{~d} t \int_{t-\varepsilon}^{t} \mathrm{~d} s \delta^{2 m}(\varepsilon) \leq \mathrm{cst} \cdot \varepsilon^{-1} \delta^{2 m}(\varepsilon)
\end{aligned}
$$

where cst denotes a constant (here depending only on $\delta$ and $m$ ) whose value may change in the remainder of the article's proofs. The hypothesis on $\delta^{2}$ implies that the above converges to 0 as $\varepsilon$ tends to 0 . 
Step 2. Small $t$ term. The term for $t \in[0, \varepsilon]$ and any $s \in[0, t]$ can be dealt with similarly, and is of a smaller order than the one in Step 1. Specifically we have

$$
\left|J_{j, S}\right|:=\varepsilon^{-2} \delta^{4 j}(\varepsilon) c_{j}\left|\int_{0}^{\varepsilon} \mathrm{d} t \int_{0}^{t} \mathrm{~d} s \Theta^{\varepsilon}(s, t)^{m-2 j}\right| \leq \varepsilon^{-2} \delta^{4 j}(\varepsilon) c_{j} \delta^{2(m-2 j)}(\varepsilon) \varepsilon^{2}=c_{j} \delta^{2 m}(\varepsilon),
$$

which converges to 0 like $o(\varepsilon)$.

Step 3. Off-diagonal. By stationarity, from (3.1), for any $s, t$ in the $\varepsilon$-off diagonal set $O D_{\varepsilon}:=$ $\{0 \leq s<t-\varepsilon<t \leq T\}$,

$$
\begin{aligned}
\Theta^{\varepsilon}(s, t) & =\left(\delta^{2}(t-s+\varepsilon)-\delta^{2}(t-s)\right)-\left(\delta^{2}(t-s)-\delta^{2}(t-s-\varepsilon)\right) \\
& =\int_{t-s}^{t-s+\varepsilon} \mathrm{d} \delta^{2}(r)-\int_{t-s-\varepsilon}^{t-s} \mathrm{~d} \delta^{2}(r) .
\end{aligned}
$$

By the concavity of $\delta^{2}$, we see that $\Theta^{\varepsilon}(s, t)$ is negative in $O D_{\varepsilon}$. According to Lemma 3.1, the off-diagonal portion $\sum_{j=0}^{(m-1) / 2} J_{j, O D_{\varepsilon}}$ of $\mathbf{E}\left[\left([X, m]_{\varepsilon}(T)\right)^{2}\right]$ is precisely equal to,

$$
\sum_{j=0}^{(m-1) / 2} J_{j, O D_{\varepsilon}}:=\sum_{j=0}^{(m-1) / 2} \varepsilon^{-2} \delta^{4 j}(\varepsilon) c_{j} \int_{\varepsilon}^{T} \mathrm{~d} t \int_{0}^{t-\varepsilon} \mathrm{d} s \Theta^{\varepsilon}(s, t)^{m-2 j} .
$$

The negativity of $\Theta^{\varepsilon}$ on $O D_{\varepsilon}$, odd power $m-2 j$, and positivity of all other factors above implies that the entire off-diagonal contribution to $\mathbf{E}\left[\left([X, m]_{\varepsilon}(T)\right)^{2}\right]$ is negative. Combining this with the results of Steps 1 and 2 , we obtain that

$$
\mathbf{E}\left[\left([X, m]_{\varepsilon}(T)\right)^{2}\right] \leq \operatorname{cst} \cdot \varepsilon^{-1} \delta^{2 m}(2 \varepsilon)
$$

which implies the sufficient condition in the theorem.

Step 4. Necessary condition. The proof of this part is more delicate than the above: it requires an excellent control of the off-diagonal term, since it is negative and turns out to be of the same order of magnitude as the diagonal term. We spell out the proof here for $m=3$. The general case is similar, and is left to the reader.

Step 4.1. Positive representation. The next elementary lemma (see the product formula in [30] (Prop. 1.1.3), or ([23], Thm. 9.6.9)) uses the following chaos integral notation: for any $n \in \mathbf{N}$, for $g \in L^{2}\left([0, T]^{n}\right), g$ symmetric in its $n$ variables, then $I_{n}(g)$ is the multiple Wiener integral of $g$ over $[0, T]^{n}$ with respect to $W$.

Lemma 3.7. Let $f \in L^{2}([0, T])$. Then $I_{1}(f)^{3}=3|f|_{L^{2}([0, T])}^{2} I_{1}(f)+I_{3}(f \otimes f \otimes f)$

Using this lemma, as well as definitions (1.3) and (2.1), recalling the notation $\Delta G_{s}(u):=G(s+\varepsilon, u)-G(s, u)$ already used in Lemma 3.3, and exploiting the fact that the covariance of two multiple Wiener integrals of different orders is 0 , we can write

$$
\begin{aligned}
\mathbf{E}\left[\left([X, 3]_{\varepsilon}(T)\right)^{2}\right]= & \frac{9}{\varepsilon^{2}} \int_{0}^{T} \mathrm{~d} s \int_{0}^{T} \mathrm{~d} t \mathbf{E}\left[I_{1}\left(\Delta G_{s}\right) I_{1}\left(\Delta G_{t}\right)\right]\left|\Delta G_{s}\right|_{L^{2}([0, T])}^{2}\left|\Delta G_{t}\right|_{L^{2}([0, T])}^{2} \\
& +\frac{1}{\varepsilon^{2}} \int_{0}^{T} \mathrm{~d} s \int_{0}^{T} \mathrm{~d} t \mathbf{E}\left[I_{3}\left(\left(\Delta G_{s}\right)^{\otimes 3}\right) I_{3}\left(\left(\Delta G_{t}\right)^{\otimes 3}\right)\right]
\end{aligned}
$$


Now we use the fact that $\mathbf{E}\left[I_{3}(h) I_{3}(\ell)\right]=\langle h, \ell\rangle_{L^{2}\left([0, T]^{3}\right)}$, plus the fact that in our stationary situation $\left|\Delta G_{s}\right|_{L^{2}([0, T])}^{2}=\delta^{2}(\varepsilon)$ for any $s$. Hence the above equals

$$
\begin{aligned}
& \frac{9 \delta^{4}(\varepsilon)}{\varepsilon^{2}} \int_{0}^{T} \mathrm{~d} s \int_{0}^{T} \mathrm{~d} t\left\langle\Delta G_{s}, \Delta G_{t}\right\rangle_{L^{2}([0, T])}+\frac{1}{\varepsilon^{2}} \int_{0}^{T} \mathrm{~d} s \int_{0}^{T} \mathrm{~d} t\left\langle\left(\Delta G_{s}\right)^{\otimes 3},\left(\Delta G_{t}\right)^{\otimes 3}\right\rangle_{L^{2}\left([0, T]^{3}\right)} \\
& =\frac{9 \delta^{4}(\varepsilon)}{\varepsilon^{2}} \int_{0}^{T} \mathrm{~d} s \int_{0}^{T} \mathrm{~d} t \int_{0}^{T} \mathrm{~d} u \Delta G_{s}(u) \Delta G_{t}(u)+\frac{1}{\varepsilon^{2}} \int_{0}^{T} \mathrm{~d} s \int_{0}^{T} \mathrm{~d} t \iiint_{[0, T]^{3}}^{3}\left(\prod_{i=1}^{3}\left(\mathrm{~d} u_{i} \Delta G_{s}\left(u_{i}\right) \Delta G_{t}\left(u_{i}\right)\right)\right. \\
& =\frac{9 \delta^{4}(\varepsilon)}{\varepsilon^{2}} \int_{0}^{T} \mathrm{~d} u\left|\int_{0}^{T} \mathrm{~d} s \Delta G_{s}(u)\right|^{2}+\frac{1}{\varepsilon^{2}} \iiint_{[0, T]^{3}} \mathrm{~d} u_{1} \mathrm{~d} u_{2} \mathrm{~d} u_{3}\left|\int_{0}^{T} \mathrm{~d} s \prod_{i=1}^{3}\left(\Delta G_{s}\left(u_{i}\right)\right)\right|^{2} .
\end{aligned}
$$

Step 4.2. $J_{1}$ as a lower bound. The above representation is extremely useful because it turns out, as one readily checks, that of the two summands in the last expression above, the first is what we called $J_{1}$ and the second is $J_{0}$, and we can now see that both these terms are positive, which was not at all obvious before, since, as we recall, the off-diagonal contribution to either term is negative by our concavity assumption. Nevertheless, we may now have a lower bound on the $\varepsilon$-variation by finding a lower bound for the term $J_{1}$ alone. Reverting to our method of separating diagonal and off-diagonal terms, and recalling by Step 2 that we can restrict $t \geq \varepsilon$, we have

$$
\begin{aligned}
J_{1} & =\frac{9 \delta^{4}(\varepsilon)}{\varepsilon^{2}} 2 \int_{\varepsilon}^{T} \mathrm{~d} t \int_{0}^{t} \mathrm{~d} s \int_{0}^{T} \mathrm{~d} u \Delta G_{s}(u) \Delta G_{t}(u)=\frac{9 \delta^{4}(\varepsilon)}{\varepsilon^{2}} 2 \int_{\varepsilon}^{T} \mathrm{~d} t \int_{0}^{t} \mathrm{~d} s \Theta_{\varepsilon}(s, t) \\
& =\frac{9 \delta^{4}(\varepsilon)}{\varepsilon^{2}} \int_{\varepsilon}^{T} \mathrm{~d} t \int_{0}^{t} \mathrm{~d} s\left(\delta^{2}(t-s+\varepsilon)-\delta^{2}(t-s)-\left(\delta^{2}(t-s)-\delta^{2}(|t-s-\varepsilon|)\right)\right) \\
& =J_{1, D}+J_{1, O D}
\end{aligned}
$$

where, performing the change of variables $t-s \mapsto s$

$$
\begin{aligned}
J_{1, D} & :=\frac{9 \delta^{4}(\varepsilon)}{\varepsilon^{2}} \int_{\varepsilon}^{T} \mathrm{~d} t \int_{0}^{\varepsilon} \mathrm{d} s\left(\delta^{2}(s+\varepsilon)-\delta^{2}(s)-\left(\delta^{2}(s)-\delta^{2}(\varepsilon-s)\right)\right) \\
J_{1, O D} & :=\frac{9 \delta^{4}(\varepsilon)}{\varepsilon^{2}} \int_{\varepsilon}^{T} \mathrm{~d} t \int_{\varepsilon}^{t} \mathrm{~d} s\left(\delta^{2}(s+\varepsilon)-\delta^{2}(s)-\left(\delta^{2}(s)-\delta^{2}(s-\varepsilon)\right)\right) .
\end{aligned}
$$

Step 4.3. Upper bound on $\left|J_{1, O D}\right|$. We rewrite the planar increments of $\delta^{2}$ as in (3.4) to show what cancellations occur: with the change of variable $s^{\prime}:=t-s-\varepsilon$, we get $-\Theta^{\varepsilon}(s, t)=-\int_{s^{\prime}}^{s^{\prime}+\varepsilon} \mathrm{d} \delta^{2}(r)+\int_{s^{\prime}-\varepsilon}^{s^{\prime}} \mathrm{d} \delta^{2}(r)$, and

$$
\begin{aligned}
\int_{\varepsilon}^{T} \mathrm{~d} t \int_{0}^{t-\varepsilon} \mathrm{d} s\left(-\Theta^{\varepsilon}(s, t)\right) & =\int_{\varepsilon}^{T} \mathrm{~d} t\left[\int_{\varepsilon}^{t} \mathrm{~d} s^{\prime} \int_{s^{\prime}-\varepsilon}^{s^{\prime}} \mathrm{d} \delta^{2}(r)-\int_{\varepsilon}^{t} \mathrm{~d} s^{\prime} \int_{s^{\prime}}^{s^{\prime}+\varepsilon} \mathrm{d} \delta^{2}(r)\right] \\
& =\int_{\varepsilon}^{T} \mathrm{~d} t\left[\int_{0}^{t-\varepsilon} \mathrm{d} s^{\prime \prime} \int_{s^{\prime \prime}}^{s^{\prime \prime}+\varepsilon} \mathrm{d} \delta^{2}(r)-\int_{\varepsilon}^{t} \mathrm{~d} s^{\prime} \int_{s^{\prime}}^{s^{\prime}+\varepsilon} \mathrm{d} \delta^{2}(r)\right] \\
& =\int_{\varepsilon}^{T} \mathrm{~d} t\left[\int_{0}^{\varepsilon} \mathrm{d} s^{\prime \prime} \int_{s^{\prime \prime}}^{s^{\prime \prime}+\varepsilon} \mathrm{d} \delta^{2}(r)-\int_{t-\varepsilon}^{t} \mathrm{~d} s^{\prime} \int_{s^{\prime}}^{s^{\prime}+\varepsilon} \mathrm{d} \delta^{2}(r)\right]
\end{aligned}
$$

where we also used the change $s^{\prime \prime}:=s^{\prime}-\varepsilon$. Thus we have

$$
J_{1, O D}=\frac{9 \delta^{4}(\varepsilon)}{\varepsilon^{2}} \int_{\varepsilon}^{T} \mathrm{~d} t\left[\int_{t-\varepsilon}^{t} \mathrm{~d} s \int_{s}^{s+\varepsilon} \mathrm{d} \delta^{2}(r)-\int_{0}^{\varepsilon} \mathrm{d} s \int_{s}^{s+\varepsilon} \mathrm{d} \delta^{2}(r)\right]=: K_{1}+K_{2} .
$$


We can already see that $K_{1} \geq 0$ and $K_{2} \leq 0$, so it is only necessary to find an upper bound on $\left|K_{2}\right|$; by Fubini on $(r, s)$, the integrand in $K_{2}$ is calculated as

$$
\int_{0}^{\varepsilon} \mathrm{d} s \int_{s}^{s+\varepsilon} \mathrm{d} \delta^{2}(r)=-\int_{0}^{\varepsilon} \delta^{2}(r) \mathrm{d} r+\int_{\varepsilon}^{2 \varepsilon} \delta^{2}(r) \mathrm{d} r .
$$

In particular, because $\left|K_{1}\right| \ll\left|K_{2}\right|$ and $\delta^{2}$ is increasing, we get

$$
\left|J_{1, O D}\right| \leq \frac{9(T-\varepsilon) \delta^{4}(\varepsilon)}{\varepsilon^{2}}\left(\int_{\varepsilon}^{2 \varepsilon} \delta^{2}(r) \mathrm{d} r-\int_{0}^{\varepsilon} \delta^{2}(r) \mathrm{d} r\right) .
$$

Step 4.4. Lower bound on $J_{1, D}$. Note first that

$$
\int_{0}^{\varepsilon} \mathrm{d} s\left(\delta^{2}(s)-\delta^{2}(\varepsilon-s)\right)=\int_{0}^{\varepsilon} \mathrm{d} s \delta^{2}(s)-\int_{0}^{\varepsilon} \mathrm{d} s \delta^{2}(\varepsilon-s)=0 .
$$

Therefore

$$
J_{1, D}=\frac{9 \delta^{4}(\varepsilon)}{\varepsilon^{2}} \int_{\varepsilon}^{T} \mathrm{~d} t \int_{0}^{\varepsilon} \mathrm{d} s\left(\delta^{2}(s+\varepsilon)-\delta^{2}(s)\right)=\frac{9 \delta^{4}(\varepsilon)}{\varepsilon^{2}}(T-\varepsilon) \int_{0}^{\varepsilon} \mathrm{d} s \int_{s}^{s+\varepsilon} \mathrm{d} \delta^{2}(r) .
$$

We can also perform a Fubini on the integral in $J_{1, D}$, easily obtaining

$$
J_{1, D}=\frac{9 \delta^{4}(\varepsilon)}{\varepsilon^{2}}(T-\varepsilon)\left(\varepsilon \delta^{2}(2 \varepsilon)-\int_{0}^{\varepsilon} \delta^{2}(r) \mathrm{d} r\right) .
$$

Step 4.5. Conclusion. We may now compare $J_{1, D}$ and $\left|J_{1, O D}\right|$ : by the results of Steps 4.1 and 4.2 ,

$$
\begin{aligned}
J_{1}= & J_{1, D}-\left|J_{1, O D}\right| \geq \frac{9 \delta^{4}(\varepsilon)}{\varepsilon^{2}}(T-\varepsilon)\left(\varepsilon \delta^{2}(2 \varepsilon)-\int_{0}^{\varepsilon} \delta^{2}(r) \mathrm{d} r\right) \\
& -\frac{9 \delta^{4}(\varepsilon)}{\varepsilon^{2}}(T-\varepsilon)\left(\int_{\varepsilon}^{2 \varepsilon} \delta^{2}(r) \mathrm{d} r-\int_{0}^{\varepsilon} \delta^{2}(r) \mathrm{d} r\right)=\frac{9 \delta^{4}(\varepsilon)}{\varepsilon^{2}}(T-\varepsilon) \int_{\varepsilon}^{2 \varepsilon}\left(\delta^{2}(2 \varepsilon)-\delta^{2}(r)\right) \mathrm{d} r .
\end{aligned}
$$

When $\delta$ is in the Hölder scale $\delta(r)=r^{H}$, the above quantity is obviously commensurate with $\delta^{6}(\varepsilon) / \varepsilon$, which implies the desired result, but in order to be sure we are treating all cases, we now present a general proof which only relies on the fact that $\delta^{2}$ is increasing and concave.

Below we use the notation $\left(\delta^{2}\right)^{\prime}$ for the density of $\mathrm{d} \delta^{2}$, which exists a.e. since $\delta^{2}$ is concave. The mean value theorem and the concavity of $\delta^{2}$ then imply that for any $r \in[\varepsilon, 2 \varepsilon]$,

$$
\delta^{2}(2 \varepsilon)-\delta^{2}(r) \geq(2 \varepsilon-r) \inf _{[\varepsilon, 2 \varepsilon]}\left(\delta^{2}\right)^{\prime}=(2 \varepsilon-r)\left(\delta^{2}\right)^{\prime}(2 \varepsilon)
$$

Thus we can write

$$
\begin{aligned}
J_{1} & \geq 9(T-\varepsilon) \varepsilon^{-1} \delta^{4}(\varepsilon)\left(\delta^{2}\right)^{\prime}(2 \varepsilon) \int_{\varepsilon}^{2 \varepsilon}(2 \varepsilon-r) \mathrm{d} r=9(T-\varepsilon) \varepsilon^{-1} \delta^{4}(\varepsilon)\left(\delta^{2}\right)^{\prime}(2 \varepsilon) \varepsilon^{2} / 2 \\
& \geq \operatorname{cst} \cdot \delta^{4}(\varepsilon) \cdot\left(\delta^{2}\right)^{\prime}(2 \varepsilon) .
\end{aligned}
$$

Since $\delta^{2}$ is concave, and $\delta(0)=0$, we have $\delta^{2}(\varepsilon) \geq \delta^{2}(2 \varepsilon) / 2$. Hence, with the notation $f(x)=\delta^{2}(2 x)$, we have

$$
J_{1} \geq \operatorname{cst} \cdot f^{2}(\varepsilon) f^{\prime}(\varepsilon)=\operatorname{cst} \cdot\left(f^{3}\right)^{\prime}(\varepsilon) .
$$


Therefore we have that $\lim _{\varepsilon \rightarrow 0}\left(f^{3}\right)^{\prime}(\varepsilon)=0$. We prove this implies $\lim _{\varepsilon \rightarrow 0} \varepsilon^{-1} f^{3}(\varepsilon)=0$. Indeed, fix $\eta>0$; then there exists $\varepsilon_{\eta}>0$ such that for all $\varepsilon \in\left(0, \varepsilon_{\eta}\right], 0 \leq\left(f^{3}\right)^{\prime}(\varepsilon) \leq \eta$ (we used the positivity of $\left.\left(\delta^{2}\right)^{\prime}\right)$. Hence, also using $f(0)=0$, for any $\varepsilon \in\left(0, \varepsilon_{\eta}\right]$,

$$
0 \leq \frac{f^{3}(\varepsilon)}{\varepsilon}=\frac{1}{\varepsilon} \int_{0}^{\varepsilon}\left(f^{3}\right)^{\prime}(x) \mathrm{d} x \leq \frac{1}{\varepsilon} \int_{0}^{\varepsilon} \eta \mathrm{d} x=\eta .
$$

This proves that $\lim _{\varepsilon \rightarrow 0} \varepsilon^{-1} f^{3}(\varepsilon)=0$, which is equivalent to the announced necessary condition, and finishes the proof of the theorem.

\subsection{Non-stationary case}

The concavity and stationarity assumptions were used heavily above for the proof of the necessary condition in Theorem 3.6. The next result, proved in the Appendix, shows they can be considerably weakened while still resulting in a sufficient condition: we only need a weak uniformity condition on the variances, coupled with a natural bound on the second-derivative measure of $\delta^{2}$.

Theorem 3.8. Let $m>1$ be an odd integer. Let $X$ be a centered Gaussian process on $[0, T]$ with canonical metric

$$
\delta^{2}(s, t):=\mathbf{E}\left[(X(t)-X(s))^{2}\right] .
$$

Define a univariate function on $[0, T]$, also denoted by $\delta^{2}$, via

$$
\delta^{2}(r):=\sup _{s \in[0, T]} \delta^{2}(s, s+r),
$$

and assume that for $r$ near 0 ,

$$
\delta(r)=o\left(r^{1 / 2 m}\right) .
$$

Assume that, in the sense of distributions, the derivative $\partial \delta^{2} /(\partial s \partial t)$ is a signed $\sigma$-finite measure $\mu$ on $[0, T]^{2}-\Delta$ where $\Delta$ is the diagonal $\{(s, s) \mid s \in[0, T]\}$. Denote the off-diagonal simplex by $O D=\{(s, t): 0 \leq s \leq t-\varepsilon \leq T\}$; assume $\mu$ satisfies, for some constant $c$ and for all $\varepsilon$ small enough,

$$
|\mu|(O D) \leq c \varepsilon^{-(m-1) / m},
$$

where $|\mu|$ is the total variation measure of $\mu$. Then $X$ has zero odd mth variation.

Example 3.9. A typical situation covered by the above theorem is that of the Riemann-Liouville fBm $B^{H, R L}$ and similar non-stationary processes. The process $B^{H, R L}$ is defined by $B^{H, R L}(t)=\int_{0}^{t}(t-s)^{H-1 / 2} \mathrm{~d} W(s)$; it differs from the standard $\mathrm{fBm}$ by a bounded variation process, and as such it has zero odd $m$ th variation for any $H>1 /(2 m)$. This can also be obtained via our theorem, because $B^{H, R L}$ is a member of the class of Gaussian processes whose canonical metric satisfies

$$
|t-s|^{H} \leq \delta(s, t) \leq 2|t-s|^{H} .
$$

(see [25]). For any process satisfying (3.8), our theorem's condition on variances is equivalent to $H>1 /(2 m)$, while for the other condition, a direct computation yields $\mu(\mathrm{d} s \mathrm{~d} t) \asymp|t-s|^{2 H-2} \mathrm{~d} s \mathrm{~d} t$ off the diagonal, and therefore, for $H<1 / 2$,

$$
\mu(O D)=|\mu|(O D) \asymp \int_{0}^{T} \int_{\varepsilon}^{t} s^{2 H-2} \mathrm{~d} s \mathrm{~d} t \asymp \varepsilon^{2 H-1},
$$

where the notation " $x \asymp y$ " means $x / y$ is bounded above and below by positive constants. This quantity is bounded above by $\varepsilon^{-1+1 / m}$ as soon as $H \geq 1 /(2 m)$, of course, so the strict inequality is sufficient to apply the theorem and conclude that $B^{H, R L}$ all other processes satisfying (3.8) have zero odd $m$ th variation. 
Example 3.10. One can generalize Example 3.9 to any Gaussian process with a Volterra-convolution kernel: let $\gamma^{2}$ be a univariate increasing concave function, differentiable everywhere except possibly at 0 , and define

$$
X(t)=\int_{0}^{t}\left(\frac{\mathrm{d} \gamma^{2}}{\mathrm{~d} r}\right)^{1 / 2}(t-r) \mathrm{d} W(r) .
$$

Then one can show (see [25]) that the canonical metric $\delta^{2}(s, t)$ of $X$ is bounded above by $2 \gamma^{2}(|t-s|)$, so that we can use the univariate $\delta^{2}=2 \gamma^{2}$, and also $\delta^{2}(s, t)$ is bounded below by $\gamma^{2}(|t-s|)$. Similar calculations to the above then easily show that $X$ has zero odd $m$ th variation as soon as $\delta^{2}(r)=o\left(r^{1 /(2 m)}\right)$. Hence there are processes with non stationary increments that are more irregular than fractional Brownian for any $H>1 /(2 m)$ which still have zero odd $m$ th variation: use for instance the $X$ above with $\gamma^{2}(r)=r^{1 /(2 m)} / \log (1 / r)$.

\section{Non-Gaussian CASE}

Now assume that $X$ is given by (1.3) and $M$ is a square-integrable (non-Gaussian) continuous martingale, $m$ is an odd integer, and define a positive non-random measure $\mu$ for $\bar{s}=\left(s_{1}, s_{2}, \ldots, s_{m}\right) \in[0, T]^{m}$ by:

$$
\mu(d \bar{s})=\mu\left(\mathrm{d} s_{1} \mathrm{~d} s_{2} \ldots \mathrm{d} s_{m}\right)=\mathbf{E}\left[\mathrm{d}[M]\left(s_{1}\right) \mathrm{d}[M]\left(s_{2}\right) \ldots \mathrm{d}[M]\left(s_{m}\right)\right],
$$

where $[M]$ is the quadratic variation process of $M$. We make the following assumption on $\mu$.

(A) The non-negative measure $\mu$ is absolutely continuous with respect to the Lebesgue measure $d \bar{s}$ on $[0, T]^{m}$ and

$K(\bar{s}):=\mathrm{d} \mu / d \bar{s}$ is bounded by a tensor-power function: $0 \leq K\left(s_{1}, s_{2}, \ldots, s_{m}\right) \leq \Gamma^{2}\left(s_{1}\right) \Gamma^{2}\left(s_{2}\right) \ldots \Gamma^{2}\left(s_{m}\right)$ for some non-negative function $\Gamma$ on $[0, T]$.

A large class of processes satisfying (A) is the case where $M(t)=\int_{0}^{t} H(s) \mathrm{d} W(s)$ where $H \in L^{2}([0, T] \times \Omega)$ and $W$ is a standard Wiener process, and we assume $\mathbf{E}\left[H^{2 m}(t)\right]$ is finite for all $t \in[0, T]$. Indeed then by Hölder's inequality, since we can take $K(\bar{s})=\mathbf{E}\left[H^{2}\left(s_{1}\right) H^{2}\left(s_{2}\right) \ldots H^{2}\left(s_{m}\right)\right]$, we see that $\Gamma(t)=\left(\mathbf{E}\left[H^{2 m}(t)\right]\right)^{1 /(2 m)}$ works.

We will show that the sufficient conditions for zero odd variation in the Gaussian cases generalize to the case of condition (A), by associating $X$ with the Gaussian process

$$
Z(t):=\int_{0}^{T} \tilde{G}(t, s) \mathrm{d} W(s),
$$

where $\tilde{G}(t, s):=\Gamma(s) G(t, s)$. We have the following result (see proof in the Appendix).

Theorem 4.1. Let $m$ be an odd integer $\geq 3$. Let $X$ and $Z$ be as defined in (1.3) and (4.2). Assume $M$ satisfies condition (A) and $Z$ is well-defined and satisfies the hypotheses of Theorem 3.6 or Theorem 3.8 relative to a univariate function $\delta$. Assume that for some constant $c>0$, and every small $\varepsilon>0$,

$$
\int_{t=2 \varepsilon}^{T} \mathrm{~d} t \int_{s=0}^{t-2 \varepsilon} \mathrm{d} s \int_{u=0}^{T}\left|\Delta \tilde{G}_{t}(u)\right|\left|\Delta \tilde{G}_{s}(u)\right| \mathrm{d} u \leq c \varepsilon \delta^{2}(2 \varepsilon),
$$

where we use the notation $\Delta \tilde{G}_{t}(u)=\tilde{G}(t+\varepsilon, u)-\tilde{G}(t, u)$. Then $X$ has zero odd $m$ th variation.

The next proposition, whose proof is in the Appendix, illustrates the range of applicability of Theorem 4.1. We will use it to construct classes of examples of martingale-based processes $X$ to which the theorem applies.

Proposition 4.2. Let $X$ be defined by (1.3). Assume $m$ is an odd integer $\geq 3$ and condition (A) holds. Assume that $\tilde{G}(t, s):=\Gamma(s) G(t, s)$ can be bounded above as follows: for all $s, t$,

$$
\tilde{G}(t, s)=\mathbf{1}_{s \leq t} g(t, s)=\mathbf{1}_{s \leq t}|t-s|^{1 /(2 m)-1 / 2} \tilde{f}(t, s)
$$


in which the bivariate function $\tilde{f}$ is positive and bounded as

$$
|\tilde{f}(t, s)| \leq f(|t-s|)
$$

where the univariate function $f$ is increasing, and concave on $\mathbf{R}_{+}$, with $\lim _{r \rightarrow 0} f(r)=0$, and where $g$ has a second mixed derivative such that

$$
\begin{aligned}
\left|\frac{\partial g}{\partial t}(t, s)\right|+\left|\frac{\partial g}{\partial s}(t, s)\right| & \leq c|t-s|^{1 /(2 m)-3 / 2} ; \\
\left|\frac{\partial^{2} g}{\partial s \partial t}(t, s)\right| & \leq c|t-s|^{1 /(2 m)-5 / 2} .
\end{aligned}
$$

Assume $t \mapsto g(s, t)$ is decreasing and $t \mapsto \tilde{f}(s, t)$ is increasing. Then $X$ has zero odd $m$-variation.

The presence of the indicator function $\mathbf{1}_{s \leq t}$ in the expression for $\tilde{G}$ above is typical of most models, since it coincides with asking that $Z$ be adapted to the filtrations of $W$, which is equivalent to $X$ being adapted to the filtration of $M$. The proposition covers a wide variety of martingale-based models, which can be quite far from Gaussian models in the sense that they can have only a few moments. We describe one easily constructed class.

Example 4.3. Assume that $M$ is a martingale such that $\mathbf{E}\left[|\mathrm{d}[M] / \mathrm{d} t|^{m}\right]$ is bounded above by a constant $c^{2 m}$ uniformly in $t \leq T$. For instance we can take $M_{t}=\int_{0}^{t} H_{s}(s) \mathrm{d} W(s)$ where $H$ is a $W$-adapted process with $\mathbf{E}\left[\left|H_{s}\right|^{2 m}\right]^{1 / 2 m} \leq c$. This boundedness assumption implies that we can take $\Gamma \equiv c$ in condition (A), and $\tilde{G}=c G$. Let $G(t, s)=G_{R L f B m}(t, s):=\mathbf{1}_{s \leq t}|t-s|^{1 /(2 m)-1 / 2+\alpha}$ for some $\alpha>0$; in other words, $G$ is the Brownian representation kernel of the Riemann-Liouville $\mathrm{fBm}$ with parameter $H=1 /(2 m)+\alpha>1 /(2 m)$. It is immediate to check that the assumptions of Proposition 4.2 are satisfied for this class of martingale-based models, which implies that the corresponding $X$ defined by (1.3) have zero odd $m$ th variation.

More generally, assume that $G$ is bounded above by a multiple of $G_{R L f B m}$, and assume the two partial derivatives of $G$, and the mixed second order derivative of $G$, are bounded by the corresponding (multiples of) derivatives of $G_{R L f B m}$; one can check that the standard fBm's kernel is in this class, and that the martingalebased models of this class also satisfy the assumptions of Proposition 4.2, resulting again zero odd $m$ th variations for the corresponding $X$ defined in (1.3). For the sake of conciseness, we will omit the details, which are tedious and straightforward.

The main quantitative assumption on the univariate function $\delta(\varepsilon)$ corresponding to $\tilde{G}$ in the theorem, i.e. $\delta(r)=o\left(r^{1 /(2 m)}\right)$, can be reinterpreted as a regularity condition on $X$.

Example 4.4. For example, if $X$ has fractional exponential moments, in the sense that for some constants $a>0$ and $0<\beta \leq 2, \mathbf{E}\left[\exp \left(a|X(t)-X(s)|^{\beta}\right)\right]$ is finite for all $s, t$, then an almost-sure uniform modulus of continuity for $X$ is $r \mapsto \delta(r) \log ^{\beta / 2}(1 / r)$. This can be established by using Corollary 4.5 in [39]. By using the Burkholder-Davis-Gundy inequality on the exponential martingale based on $M$, we can prove that such fractional exponential moments hold, for instance, in the setting of Example 4.3, if there exists $b>0$ such that $\mathbf{E}\left[\exp \left(b\left|H_{s}\right|^{2 \beta}\right)\right]$ is bounded in $s \in[0, T]$. If one only has standard (non-exponential) moments, similar (less sharp) results can be obtained via Kolmogorov's continuity criterion instead of [39]. All details are left to the reader. 


\section{Stochastic calculus}

This section's goal is to define the so-called symmetric stochastic integral and its associated Itô's formula for processes which are not fBm. The reader may refer to the Introduction (Sect. 1) for motivations on why we study this topic. We concentrate on Gaussian processes under hypotheses similar to those used in Section 3.3 (Thm. 3.8). The basic strategy is to use the results of [20] which were applied to fBm. Let $X$ be a stochastic process on $[0,1]$. According to Sections 3 and 4 in [20] (specifically, according to the proof of part 1 of Theorem 4.4 therein), if for every bounded measurable function $g$ on $\mathbf{R}$, the limit

$$
\lim _{\varepsilon \rightarrow 0} \frac{1}{\varepsilon} \int_{0}^{1} \mathrm{~d} u\left(X_{u+\varepsilon}-X_{u}\right)^{m} g\left(\frac{X_{u+\varepsilon}+X_{u}}{2}\right)=0
$$

holds in probability, for both $m=3$ and $m=5$, then for every $t \in[0,1]$ and every $f \in C^{6}(\mathbf{R})$, the symmetric ("generalized Stratonovich") stochastic integral

$$
\int_{0}^{t} f^{\prime}\left(X_{u}\right) d^{\circ} X_{u}=: \lim _{\varepsilon \rightarrow 0} \frac{1}{\varepsilon} \int_{0}^{t} \mathrm{~d} u\left(X_{u+\varepsilon}-X_{u}\right) \frac{1}{2}\left(f^{\prime}\left(X_{u+\varepsilon}\right)+f^{\prime}\left(X_{u}\right)\right)
$$

exists and we have the Itô's formula

$$
f\left(X_{t}\right)=f\left(X_{0}\right)+\int_{0}^{t} f^{\prime}\left(X_{u}\right) d^{\circ} X_{u} .
$$

Our goal is thus to prove (5.1) for a wide class of Gaussian processes $X$, which will in turn imply the existence of (5.2) and the Itô's formula (5.3).

If $X$ has stationary increments in the sense of Section 3.2, meaning that $\mathbf{E}\left[\left(X_{s}-X_{t}\right)^{2}\right]=\delta^{2}(t-s)$ for some univariate canonical metric function $\delta$, then by using $g \equiv \mathbf{1}$ and our Theorem 3.6, we see that for (5.1) to hold, we must have $\delta(r)=o\left(r^{1 / 6}\right)$. If one wishes to treat non-stationary cases, we notice that (5.1) for $g \equiv 1$ is the result of our non-stationary Theorem 3.8, so it is necessary to use that theorem's hypotheses, which include the non-stationary version of $\delta(r)=o\left(r^{1 / 6}\right)$. But we will also need some non-degeneracy conditions in order to apply the quartic linear regression method of [20]. These are conditions (i) and (ii) in the next Theorem. Condition (iii) therein is essentially a consequence of the condition that $\delta^{2}$ be increasing and concave. These conditions are all further discussed after the statement of the next theorem and its corollary.

Theorem 5.1. Let $m \geq 3$ be an odd integer. Let $X$ be a Gaussian process on $[0,1]$ satisfying the hypotheses of Theorem 3.8. This means in particular that we denote as usual its canonical metric by $\delta^{2}(s, t)$, and that there exists a univariate increasing and concave function $\delta^{2}$ such that $\delta(r)=o\left(r^{1 /(2 m)}\right)$ and $\delta^{2}(s, t) \leq \delta^{2}(|t-s|)$. Assume that for $u<v$, the functions $u \mapsto \operatorname{Var}\left[X_{u}\right]=: Q_{u}, v \mapsto \delta^{2}(u, v)$, and $u \mapsto-\delta^{2}(u, v)$ are increasing and concave. Assume there exist positive constants $a>1, b<1 / 2, c>1 / 4$, and $c^{\prime}>0$ such that for all $\varepsilon<u<v \leq 1$,

(i) $c \delta^{2}(u) \leq Q_{u}$

(ii) $c^{\prime} \delta^{2}(u) \delta^{2}(v-u) \leq Q_{u} Q_{v}-Q^{2}(u, v)$;

(iii)

$$
\frac{\delta(a u)-\delta(u)}{(a-1) u}<b \frac{\delta(u)}{u} .
$$

Then for every bounded measurable function $g$ on $\mathbf{R}$,

$$
\lim _{\varepsilon \rightarrow 0} \frac{1}{\varepsilon^{2}} \mathbf{E}\left[\left(\int_{0}^{1} \mathrm{~d} u\left(X_{u+\varepsilon}-X_{u}\right)^{m} g\left(\frac{X_{u+\varepsilon}+X_{u}}{2}\right)\right)^{2}\right]=0 .
$$


When we apply this theorem to the case $m=3$, the assumption depending on $m$, namely $\delta(r)=o\left(r^{1 /(2 m)}\right)$ is satisfied a fortiori for $m=5$ as well, which means that under the assumption $\delta(r)=o\left(r^{1 / 6}\right)$, the theorem's conclusion holds for $m=3$ and $m=5$. Therefore, as mentioned in the strategy above, we immediately get the following.

Corollary 5.2. Assume the hypotheses of Theorem 5.1 with $m=3$. We have existence of the symmetric integral in (5.2), and its Itô's formula (5.3), for every $f \in C^{6}(\mathbf{R})$ and $t \in[0,1]$.

The end of Section 3.3 contains examples satisfying the hypotheses of Theorem 3.8; most of these examples also satisfy the monotonicity and convexity conditions in the above theorem. We state this formally, omitting the details of checking the conditions.

Example 5.3. The conclusion of Corollary 5.2 applies to The Riemann-Liouville fBm described in Example 3.9, which is a Gaussian process with non-stationary increments. It also applies to any member of the wider class of processes in Example 3.10 for which the function $\gamma$ defined therein satisfies conditions (i), (ii), and (iii) of Theorem 5.1. This includes the family of processes such that $\gamma(r)=r^{H} \log ^{\beta}(1 / r)$ for $H \in(1 / 6,1)$ and $\beta \in \mathbf{R}$, the case $\beta=0$ yielding the Riemann-Liouville fBm processes.

Before proceeding to the proof of Theorem 5.1, we provide a broader discussion of its hypotheses.

Condition (i) is a type of coercivity assumption on the non-degeneracy of $X$ 's variances in comparison to its increments's variances. The hypotheses of Theorem 3.8 imply that $Q_{u} \leq \delta^{2}(u)$, and condition (i) simply adds that these two quantities should be commensurate, with a lower bound that it not too small. The "Volterra convolution"-type class of processes (3.9) given at the end of Section 3.3, which includes the Riemann-Liouville fBm's, satisfies condition (i) with $c=1 / 2$. In the stationary case, (i) is trivially satisfied since $Q_{u} \equiv \delta^{2}(u)$.

Condition (ii) is also a type of coercivity condition. It too is satisfied in the stationary case. We prove this claim, since it is not immediately obvious. In the stationary case, since $\delta^{2}(u, v)=\delta^{2}(v-u)=Q_{v-u}$, we calculate

$$
Q_{u} Q_{v}-Q^{2}(u, v)=Q_{u} Q_{v}-4^{-1}\left(Q_{u}+Q_{v}-Q_{v-u}\right)^{2}
$$

and after rearranging some terms we obtain

$$
Q_{u} Q_{v}-Q^{2}(u, v)=2^{-1} Q_{v-u}\left(Q_{u}+Q_{v}\right)-4^{-1}\left(Q_{v}-Q_{u}\right)^{2}-4^{-1} Q_{v-u}^{2}
$$

We note first that by the concavity of $Q$, we have $Q_{v}-Q_{u}<Q_{v-u}$, and consequently, $\left(Q_{v}-Q_{u}\right)^{2} \leq$ $\left(Q_{v}-Q_{u}\right) Q_{v-u} \leq Q_{v} Q_{v-u}$. This implies

$$
Q_{u} Q_{v}-Q^{2}(u, v) \geq 2^{-1} Q_{v-u} Q_{u}+4^{-1}\left(Q_{v-u} Q_{v}-Q_{v-u}^{2}\right) .
$$

Now by monotonicity of $Q$, we can write $Q_{v-u} Q_{v} \geq Q_{v-u}^{2}$. This, together with condition (i), yield condition (ii) since we now have

$$
Q_{u} Q_{v}-Q^{2}(u, v) \geq 2^{-1} Q_{v-u} Q_{u} \geq 2^{-1} c^{2} \delta^{2}(v-u) \delta^{2}(u) .
$$

Lastly, condition (iii) represents a strengthened concavity condition on the univariate function $\delta$. Indeed, the left-hand side in (5.4) is the slope of the secant of the graph of $\delta$ between the points $u$ and au, while the right-hand side is $b$ times the slope of the secant from 0 to $u$. If $b$ were allowed to be 1 , (iii) would simply be a consequence of convexity. Here taking $b \leq 1 / 2$ means that we are exploiting the concavity of $\delta^{2}$; the fact that condition (iii) requires slightly more, namely $b$ strictly less than $1 / 2$, allows us to work similarly to the scale $\delta(r)=r^{H}$ with $H<1 / 2$, as opposed to simply asking $H \leq 1 / 2$. Since the point of the theorem is to allow continuity moduli which are arbitrarily close to $r^{1 / 6}$, condition (iii) is hardly a restriction. 
Proof of Theorem 5.1.

Step 0. Setup. The expectation to be evaluated is written, as usual, as a double integral over $(u, v) \in[0,1]^{2}$. For $\varepsilon>0$ fixed, we define the "off-diagonal" set

$$
D_{\varepsilon}=\left\{(u, v) \in[0,1]^{2}: \varepsilon^{1-\rho} \leq u \leq v-\varepsilon^{1-\rho}<v \leq 1\right\}
$$

where $\rho \in(0,1)$ is fixed. Using the boundedness of $g$ and Cauchy-Schwarz's inequality, thanks to the hypothesis $\delta(r)=o\left(r^{1 /(2 m)}\right)$, the term corresponding to the diagonal part (integral over $D_{\varepsilon}^{c}$ ) can be treated identically to what was done in [20] in dealing with their term $\mathcal{J}^{\prime}(\varepsilon)$ following the statement of their Lemma 5.1, by choosing $\rho$ small enough. It is thus sufficient to prove that

$$
\mathcal{J}(\varepsilon):=\frac{1}{\varepsilon^{2}} \mathbf{E}\left[\iint_{D_{\varepsilon}} \mathrm{d} u \mathrm{~d} v\left(X_{u+\varepsilon}-X_{u}\right)^{m}\left(X_{v+\varepsilon}-X_{v}\right)^{m} g\left(\frac{X_{u+\varepsilon}+X_{u}}{2}\right) g\left(\frac{X_{v+\varepsilon}+X_{v}}{2}\right)\right]
$$

tends to 0 as $\varepsilon$ tends to 0 . We now use the same method and notation as in Step 3 of the proof of Theorem 4.1 in [20]. It proceeds through the linear regression analysis of the Gaussian vector $\left(G_{1}, G_{2}, G_{3}, G_{4}\right):=\left(X_{u+\varepsilon}+\right.$ $X_{u}, X_{v+\varepsilon}+X_{v}, X_{u+\varepsilon}-X_{u}, X_{v+\varepsilon}-X_{v}$ ). In order to avoid repeating arguments from that proof, we only state and prove the new lemmas which are required. The new elements come from the analysis of the Gaussian vector $\left(\Gamma_{3}, \Gamma_{4}\right)^{t}:=A\left(G_{1}, G_{2}\right)$ where $A:=\Lambda_{21}\left(\Lambda_{11}\right)^{-1}$ where $\Lambda_{11}$ is the covariance of the vector $\left(G_{1}, G_{2}\right)$ and $\Lambda_{21}$ is the matrix $\left\{\operatorname{Cov}\left(G_{i+2}, G_{j}\right): i, j=1,2\right\}$, as well as from the the centered Gaussian vector $\left(Z_{3}, Z_{4}\right)$ which is the component independent of $\left(G_{3}, G_{4}\right)$ in its linear regression against $\left(G_{1}, G_{2}\right)$, i.e. $\left(G_{3}, G_{4}\right)^{t}=$ $A\left(G_{1}, G_{2}\right)^{t}+\left(Z_{3}, Z_{4}\right)$.

Step 1. Translating Lemma 5.3 from [20]. Using the fact that $\mathbf{E}\left[Z_{\ell}^{2}\right] \leq \mathbf{E}\left[G_{\ell}^{2}\right] \leq \delta^{2}(\varepsilon)$, this lemma translates as the following, proved in a version of this article which can be found on arXiv: see [37].

Lemma 5.4. Let $k \geq 2$ be an integer. Then for $\ell=3,4$,

$$
\iint_{D_{\varepsilon}} \mathbf{E}\left[\left|\Gamma_{\ell}\right|^{k}\right] \mathrm{d} u \mathrm{~d} v \leq \operatorname{cst} \cdot \varepsilon \delta^{k}(\varepsilon) .
$$

Step 2. Translating Lemma 5.4 from [20]. We will prove the following result

Lemma 5.5. For all $j \in\{0,1, \ldots,(m-1) / 2\}$,

$$
\iint_{D_{\varepsilon}}\left|\mathbf{E}\left[Z_{3} Z_{4}\right]\right|^{m-2 j} \mathrm{~d} u \mathrm{~d} v \leq \operatorname{cst} \cdot \varepsilon \delta^{2(m-2 j)}(\varepsilon) .
$$

Proof of Lemma 5.5. As in [20], we have

$$
\left|\mathbf{E}\left[Z_{3} Z_{4}\right]\right|^{m-2 j} \leq \mathrm{cst} \cdot\left|\mathbf{E}\left[G_{3} G_{4}\right]\right|^{m-2 j}+\mathrm{cst} \cdot\left|\mathbf{E}\left[\Gamma_{3} \Gamma_{4}\right]\right|^{m-2 j} .
$$

The required estimate for the term corresponding to $\left.\mid \mathbf{E}\left[\Gamma_{3} \Gamma_{4}\right]\right]^{m-2 j}$ follows by Cauchy-Schwarz's inequality and Lemma 5.4. For the term corresponding to $\left|\mathbf{E}\left[G_{3} G_{4}\right]\right|^{m-2 j}$, we recognize that $\mathbf{E}\left[G_{3} G_{4}\right]$ is the negative planar increment $\Theta^{\varepsilon}(u, v)$ defined in (3.1). Thus the corresponding term was already considered in the proof of Theorem (3.8). More specifically, up to the factor $\varepsilon^{2} \delta^{-4 j}(\varepsilon)$, we now have to estimated the same integral as in Step 2 of that theorem's proof: see expression (A.2) for the term we called $J_{j, O D}$. This means that

$$
\iint_{D_{\varepsilon}}\left|\mathbf{E}\left[G_{3} G_{4}\right]\right|^{m-2 j} \mathrm{~d} u \mathrm{~d} v \leq \frac{\varepsilon^{2}}{\delta^{4 j}(\varepsilon)} J_{j, O D} \leq \varepsilon^{2}|\mu|(O D) \delta^{2(m-2 j-1)}(\varepsilon) .
$$


Our hypotheses borrowed from Theorem 3.8 that $|\mu|(O D) \leq \mathrm{cst} \cdot \varepsilon^{1 / m-1}$ and that $\delta^{2}(\varepsilon)=o\left(r^{1 /(2 m)}\right)$ now imply that the above is $\ll \varepsilon \delta^{2(m-2 j)}(\varepsilon)$, concluding the lemma's proof.

Step 4. Conclusion. The remainder of the proof of the theorem is to check that Lemmas 5.4 and 5.5 do imply the claim of the theorem; this is done exactly as in Steps 3 and 4 of the proof of Theorem 4.1 in [20]. Since such a task is only bookkeeping, we omit it, concluding the proof of Theorem 5.1.

Acknowledgements. The work of F. Russo was partially supported by the ANR Project MASTERIE 2010 BLAN-012101. The work of F. Viens is partially supported by NSF DMS grant 0907321. Constructive comments by referees and editors are gratefully acknowledged and resulted in several improvements.

\section{APPENDIX}

Proof of Lemma 3.1. The formula in the lemma is an easy consequence of the following formula, which can be found as Lemma 5.2 in [20]: for any centered jointly Gaussian pair of r.v.'s $(Y, Z)$, we have $\mathbf{E}\left[Y^{m} Z^{m}\right]=$ $\sum_{j=0}^{(m-1) / 2} c_{j} \mathbf{E}[Y Z]^{m-2 j} \operatorname{Var}[X]^{j} \operatorname{Var}[Y]^{j}$. To see that the $c_{j}$ 's are positive, note that one can decompose each odd monomial into the basis of odd-order Hermite polynomials: $x^{m}=\sum_{j=0}^{(m-1) / 2} a_{2 j+1} H_{2 j+1}(x)$, from whence it follows, thanks to the orthogonality of Hermite polynomials of Gaussian rv's, that $c_{j}=\left(a_{2 j+1}\right)^{2}$.

Proof of Lemma 3.3. The proof of this lemma is elementary. It follows from two uses of the multiplication formula for Wiener's integrals ([30], Prop. 1.1.3), for instance. All details are left to the reader.

Proof of Lemma 3.4. Reintroducing the notation $X$ and $\Theta$ into the formula in Lemma 3.3, we get

$$
\mathcal{I}_{1}=\frac{3}{\varepsilon} \int_{0}^{T} \mathrm{~d} s(X(s+\varepsilon)-X(s)) \operatorname{Var}(X(s+\varepsilon)-X(s))
$$

and therefore,

$$
\mathbf{E}\left[\left|\mathcal{I}_{1}\right|^{2}\right]=\frac{9}{\varepsilon^{2}} \int_{0}^{T} \int_{0}^{t} \mathrm{~d} t \mathrm{~d} s \Theta^{\varepsilon}(s, t) \operatorname{Var}(X(t+\varepsilon)-X(t)) \operatorname{Var}(X(s+\varepsilon)-X(s))
$$

Using the variances of $\mathrm{fBm}$, writing $H$ instead of $1 / 6$ to improve readability,

$$
\begin{aligned}
\mathbf{E}\left[\left|\mathcal{I}_{1}\right|^{2}\right] & =\frac{9}{2} \varepsilon^{-2+4 H} \int_{0}^{T} \int_{0}^{T} \mathrm{~d} t \mathrm{~d} s \operatorname{Cov}[X(t+\varepsilon)-X(t) ; X(s+\varepsilon)-X(s)] \\
& =\frac{9}{2} \varepsilon^{-2+4 H} \operatorname{Var}\left[\int_{0}^{T}(X(t+\varepsilon)-X(t)) \mathrm{d} t\right] \\
& =\frac{9}{2} \varepsilon^{-2+4 H} \operatorname{Var}\left[\int_{T}^{T+\varepsilon} X(t) \mathrm{d} t-\int_{0}^{\varepsilon} X(t) \mathrm{d} t\right] .
\end{aligned}
$$

Bounding the variance of the difference by twice the sum of the variances,

$$
\mathbf{E}\left[\left|\mathcal{I}_{1}\right|^{2}\right] \leq 9 \varepsilon^{-2+4 H}\left(\int_{T}^{T+\varepsilon} \int_{T}^{T+\varepsilon} T^{2 H} \mathrm{~d} s \mathrm{~d} t+\int_{0}^{\varepsilon} \int_{0}^{\varepsilon} \varepsilon^{2 H} \mathrm{~d} s \mathrm{~d} t\right)=O\left(\varepsilon^{4 H}\right),
$$

proving Lemma 3.4. 
Proof of Lemma 3.5. By the technique at the start of the proof of Lemma 3.4, the product formula in ([30], Prop. 1.1.3), and the covariance of $\mathrm{fBm}$, we first get

$$
\begin{aligned}
& \mathcal{I}_{3}:=\frac{6}{\varepsilon} \int_{0}^{T} \mathrm{~d} W\left(s_{3}\right) \int_{0}^{s_{3}} \mathrm{~d} W\left(s_{2}\right) \int_{0}^{s_{2}} \mathrm{~d} W\left(s_{1}\right) \int_{0}^{T}\left[\prod_{k=1}^{3} \Delta G_{s}\left(s_{k}\right)\right] \mathrm{d} s . \\
& \mathbf{E}\left[\left|\mathcal{I}_{3}\right|^{2}\right]=\frac{12}{\varepsilon^{2}} \int_{0}^{T} \int_{0}^{t} \mathrm{~d} t \mathrm{~d} s\left(\Theta^{\varepsilon}(s, t)\right)^{3} \\
&=\frac{6}{\varepsilon^{2}} \int_{0}^{T} \int_{0}^{t} \mathrm{~d} t \mathrm{~d} s\left(|t-s+\varepsilon|^{2 H}+|t-s-\varepsilon|^{2 H}-2|t-s|^{2 H}\right)^{3} .
\end{aligned}
$$

We must take care of the absolute values, i.e. of whether $\varepsilon$ is greater or less than $t-s$. We define the "off-diagonal" portion of $\mathbf{E}\left[\left|\mathcal{I}_{3}\right|^{2}\right]$ as

$$
\mathcal{O D I}_{3}:=6 \varepsilon^{-2} \int_{2 \varepsilon}^{T} \int_{0}^{t-2 \varepsilon} \mathrm{d} t \mathrm{~d} s\left(|t-s+\varepsilon|^{2 H}+|t-s-\varepsilon|^{2 H}-2|t-s|^{2 H}\right)^{3} .
$$

For $s, t$ in the integration domain for the above integral, since $\bar{t}:=t-s>2 \varepsilon$, by two iterated applications of the Mean Value Theorem for the function $x^{2 H}$ on the intervals $[\bar{t}-\varepsilon, \bar{t}]$ and $[\bar{t}, \bar{t}+\varepsilon]$,

$$
|\bar{t}+\varepsilon|^{2 H}+|\bar{t}-\varepsilon|^{2 H}-2 \bar{t}^{2 H}=2 H(2 H-1) \varepsilon\left(\xi_{1}-\xi_{2}\right) \xi^{2 H-2}
$$

for some $\xi_{2} \in[\bar{t}-\varepsilon, \bar{t}], \xi_{1} \in[\bar{t}, \bar{t}+\varepsilon]$, and $\xi \in\left[\xi_{1}, \xi_{2}\right]$, and therefore

$$
\begin{aligned}
\left|\mathcal{O D I}_{3}\right| & \leq 384 H^{3}|2 H-1|^{3} \varepsilon^{-2} \int_{2 \varepsilon}^{T} \int_{0}^{t-2 \varepsilon}\left(\varepsilon \cdot 2 \varepsilon \cdot(t-s-\varepsilon)^{2 H-2}\right)^{3} \mathrm{~d} t \mathrm{~d} s \\
& \leq \frac{384 H^{3}|2 H-1|^{3}}{5-6 H} T \varepsilon^{6 H-1}=\frac{32}{243} T .
\end{aligned}
$$

where in the last line we substituted $H=1 / 6$. Thus the "off-diagonal" term is bounded. The diagonal part of $\mathcal{I}_{3}$ is

$$
\begin{aligned}
\mathcal{D I}_{3} & :=6 \varepsilon^{-2} \int_{0}^{T} \int_{t-2 \varepsilon}^{t} \mathrm{~d} t \mathrm{~d} s\left(|t-s+\varepsilon|^{2 H}+|t-s-\varepsilon|^{2 H}-2|t-s|^{2 H}\right)^{3} \\
& =6 \varepsilon^{-1+6 H} T \int_{0}^{2} \mathrm{~d} r\left(|r+1|^{2 H}+|r-1|^{2 H}-2|r|^{2 H}\right)^{3} \mathrm{~d} r=C T,
\end{aligned}
$$

where, having substituted $H=1 / 6$, yields that $C$ is a universal constant. Thus the diagonal part $\mathcal{D} \mathcal{I}_{3}$ of $\mathbf{E}\left[\left|\mathcal{I}_{3}\right|^{2}\right]$ is constant. This proves that $\mathcal{I}_{3}$ is bounded in $L^{2}(\Omega)$, as announced. To conclude that it cannot converge in $L^{2}(\Omega)$, recall that from [20], (Thm. 4.1 part $(2)$ ), $[X, 3]_{\varepsilon}(T)=\mathcal{I}_{1}+\mathcal{I}_{3}$ converges in distribution to a nondegenerate normal law. By Lemma 3.4, $\mathcal{I}_{1}$ converges to 0 in $L^{2}(\Omega)$. Therefore, $\mathcal{I}_{3}$ converges in distribution to a non-degenerate normal law; if it also converged in $L^{2}(\Omega)$, since the 3rd Wiener chaos is closed in $L^{2}(\Omega)$, the limit would have to be in that same chaos, and thus would not have a non-degenerate normal law.

Proof of Theorem 3.8.

Step 0. Setup. Recall the result of Lemma 3.1, where now we express $\operatorname{Var}[X(t+\varepsilon)-X(t)]=\delta^{2}(t, t+\varepsilon)$ and

$$
\Theta^{\varepsilon}(s, t)=\mu([s, s+\varepsilon] \times[t, t+\varepsilon))=\int_{s}^{s+\varepsilon} \int_{t}^{t+\varepsilon} \mu(\mathrm{d} u \mathrm{~d} v) .
$$


We again separate the diagonal term from the off-diagonal term, although this time the diagonal is twice as wide: it is defined as $\{(s, t): 0 \leq t-2 \varepsilon \leq s \leq t\}$.

Step 1. Diagonal. Using Cauchy-Schwarz's inequality which implies $\left|\Theta^{\varepsilon}(s, t)\right| \leq \delta(s, s+\varepsilon) \delta(t, t+\varepsilon)$, and bounding each term $\delta(s, s+\varepsilon)$ by $\delta(\varepsilon)$, the diagonal portion of $\mathbf{E}\left[\left([X, m]_{\varepsilon}(T)\right)^{2}\right]$ can be bounded above, in absolute value, by

$$
\frac{1}{\varepsilon^{2}} \sum_{j=0}^{(m-1) / 2} c_{j} \int_{2 \varepsilon}^{T} \mathrm{~d} t \int_{t-2 \varepsilon}^{t} \mathrm{~d} s \delta^{2 m}(\varepsilon)=\mathrm{cst} \cdot \varepsilon^{-1} \delta^{2 m}(\varepsilon) .
$$

Hypothesis (3.6) implies that this converges to 0 with $\varepsilon$. The case of $t \leq 2 \varepsilon$ works equally easily.

Step 2. Off diagonal. The off-diagonal contribution is the sum for $j=0, \ldots,(m-1) / 2$ of the terms

$$
J_{j, O D}=\varepsilon^{-2} c_{j} \int_{2 \varepsilon}^{T} \mathrm{~d} t \int_{0}^{t-2 \varepsilon} \mathrm{d} s \delta^{2 j}(s, s+\varepsilon) \delta^{2 j}(t, t+\varepsilon) \Theta^{\varepsilon}(s, t)^{m-2 j}
$$

Step 2.1. Term $J_{(m-1) / 2, O D}$. This is the dominant term. Denoting $c=\left|c_{(m-1) / 2}\right|$, we have

$$
\left|J_{(m-1) / 2, O D}\right| \leq \frac{c \delta^{2 m-2}(\varepsilon)}{\varepsilon^{2}} \int_{2 \varepsilon}^{T} \mathrm{~d} t \int_{0}^{t-2 \varepsilon} \mathrm{d} s\left|\Theta^{\varepsilon}(s, t)\right|
$$

We estimate the integral, using the formula (A.1) and Fubini's theorem:

$$
\begin{aligned}
& \int_{2 \varepsilon}^{T} \mathrm{~d} t \int_{0}^{t-2 \varepsilon} \mathrm{d} s\left|\Theta^{\varepsilon}(s, t)\right|=\int_{2 \varepsilon}^{T} \mathrm{~d} t \int_{0}^{t-2 \varepsilon} \mathrm{d} s\left|\int_{s}^{s+\varepsilon} \int_{t}^{t+\varepsilon} \mu(\mathrm{d} u \mathrm{~d} v)\right| \\
& \leq \int_{2 \varepsilon}^{T} \mathrm{~d} t \int_{0}^{t-2 \varepsilon} \mathrm{d} s \int_{s}^{s+\varepsilon} \int_{t}^{t+\varepsilon}|\mu|(\mathrm{d} u \mathrm{~d} v)=\int_{2 \varepsilon}^{T+\varepsilon} \int_{0}^{v \wedge(T-\varepsilon)}|\mu|(\mathrm{d} u \mathrm{~d} v) \int_{2 \varepsilon \vee(v-\varepsilon) \vee(u+\varepsilon)}^{v \wedge T} \int_{0 \vee(u-\varepsilon)}^{u \wedge(t-2 \varepsilon)} \mathrm{d} s \mathrm{~d} t \\
& \leq \int_{2 \varepsilon}^{T+\varepsilon} \int_{0}^{v-\varepsilon}|\mu|(\mathrm{d} u \mathrm{~d} v) \int_{v-\varepsilon}^{v} \int_{u-\varepsilon}^{u} \mathrm{~d} s \mathrm{~d} t=\varepsilon^{2} \int_{2 \varepsilon}^{T+\varepsilon} \int_{0}^{v-\varepsilon}|\mu|(\mathrm{d} u \mathrm{~d} v) .
\end{aligned}
$$

Hence we have

$$
J_{(m-1) / 2, O D} \leq c \delta^{2 m-2}(\varepsilon) \int_{v=2 \varepsilon}^{T+\varepsilon} \int_{u=0}^{v-\varepsilon}|\mu|(\mathrm{d} u \mathrm{~d} v) \leq c \delta^{2 m-2}(\varepsilon)|\mu|(O D),
$$

which again converges to 0 by hypothesis as $\varepsilon$ goes to 0 .

Step 2.2. Other $J_{j, O D}$ terms. Let now $j<(m-1) / 2$. Using Cauchy-Schwarz's inequality for all but one of the $m-2 j$ factors $\Theta$ in the expression (A.2) for $J_{j, O D}$, which is allowed because $m-2 j \geq 1$ here, exploiting the bounds on the variance terms via the univariate function $\delta$, we have

$$
\left|J_{j, O D}\right| \leq \delta^{2 m-2}(\varepsilon) c_{j} \varepsilon^{-2} \int_{2 \varepsilon}^{T} \mathrm{~d} t \int_{0}^{t-2 \varepsilon} \mathrm{d} s\left|\Theta^{\varepsilon}(s, t)\right|,
$$

which is the same term we estimated in Step 2.1. This finishes the proof of the theorem.

Proof of Theorem 4.1.

Step 0. Setup. We use an expansion for powers of martingales written explicitly at Corollary 2.18 of [14]. For any integer $k \in[0,[m / 2]]$, let $\Sigma_{m}^{k}$ be the set of permutations $\sigma$ of $m-k$ defined as those for which the first $k$ terms $\sigma^{-1}(1), \sigma^{-1}(2), \ldots, \sigma^{-1}(k)$ are chosen arbitrarily and the next $m-2 k$ terms are chosen arbitrarily among the remaining integers $\{1,2, \ldots, m-k\} \backslash\left\{\sigma^{-1}(1), \sigma^{-1}(2), \ldots, \sigma^{-1}(k)\right\}$. Let $Y$ be a fixed square-integrable martingale. We define the process $Y_{\sigma, \ell}$ (denoted in the above reference by $\sigma_{Y}^{\ell}$ ) by setting, for each $\sigma \in \Sigma_{m}^{k}$ and each $\ell=1,2, \ldots, m-k$,

$$
Y_{\sigma, \ell}(t)=\left\{\begin{array}{c}
{[Y](t) \text { if } \sigma(\ell) \in\{1,2, \ldots, k\}} \\
Y(t) \text { if } \sigma(\ell) \in\{k+1, \ldots, m-k\} .
\end{array}\right.
$$


From Corollary 2.18 of [14], we then have for all $t \in[0, T]$

$$
\left(Y_{t}\right)^{m}=\sum_{k=0}^{[m / 2]} \frac{m !}{2^{k}} \sum_{\sigma \in \Sigma_{m}^{k}} \int_{0}^{t} \int_{0}^{u_{m-k}} \ldots \int_{0}^{u_{2}} \mathrm{~d} Y_{\sigma, 1}\left(u_{1}\right) \mathrm{d} Y_{\sigma, 2}\left(u_{2}\right) \ldots \mathrm{d} Y_{\sigma, m-k}\left(u_{m-k}\right) .
$$

We use this formula to evaluate

$$
[X, m]_{\varepsilon}(T)=\frac{1}{\varepsilon} \int_{0}^{T} \mathrm{~d} s(X(s+\varepsilon)-X(s))^{m}
$$

by noting that the increment $X(s+\varepsilon)-X(s)$ is the value at time $T$ of the martingale $Y_{t}:=\int_{0}^{t} \Delta G_{s}(u) \mathrm{d} M(u)$ where we set

$$
\Delta G_{s}(u):=G(s+\varepsilon, u)-G(s, u)
$$

Hence

$$
\begin{aligned}
& (X(s+\varepsilon)-X(s))^{m} \\
& =\sum_{k=0}^{[m / 2]} \frac{m !}{2^{k}} \sum_{\sigma \in \Sigma_{m}^{k}} \int_{0}^{T} \int_{0}^{u_{m-k}} \ldots \int_{0}^{u_{2}} d[M]\left(u_{\sigma(1)}\right)\left|\Delta G_{s}\left(u_{\sigma(1)}\right)\right|^{2} \ldots d[M]\left(u_{\sigma(k)}\right)\left|\Delta G_{s}\left(u_{\sigma(k)}\right)\right|^{2} \\
& \mathrm{~d} M\left(u_{\sigma(k+1)}\right) \Delta G_{s}\left(u_{\sigma(k+1)}\right) \ldots \mathrm{d} M\left(u_{\sigma(m-k)}\right) \Delta G_{s}\left(u_{\sigma(m-k)}\right) .
\end{aligned}
$$

Therefore we can write

$$
\begin{aligned}
& {[X, m]_{\varepsilon}(T)} \\
& =\frac{1}{\varepsilon} \sum_{k=0}^{[m / 2]} \frac{m !}{2^{k}} \sum_{\sigma \in \Sigma_{m}^{k}} \int_{0}^{T} \int_{0}^{u_{m-k}} \ldots \int_{0}^{u_{2}} d[M]\left(u_{\sigma(1)}\right) \ldots d[M]\left(u_{\sigma(k)}\right) \mathrm{d} M\left(u_{\sigma(k+1)}\right) \ldots \mathrm{d} M\left(u_{\sigma(m-k)}\right) \\
& {\left[\Delta G \cdot\left(u_{\sigma(k+1)}\right) ; \ldots ; \Delta G \cdot\left(u_{\sigma(m-k)}\right) ; \Delta G \cdot\left(u_{\sigma(1)}\right) ; \Delta G \cdot\left(u_{\sigma(1)}\right) ; \ldots ; \Delta G \cdot\left(u_{\sigma(k)}\right) ; \Delta G .\left(u_{\sigma(k)}\right)\right],}
\end{aligned}
$$

where we have used the notation

$$
\left[f_{1}, f_{2}, \ldots, f_{m}\right]:=\int_{0}^{T} f_{1}(s) f_{2}(s) \ldots f_{m}(s) \mathrm{d} s .
$$

To calculate the expected square of the above, we will bound it above by the sum over $k$ and $\sigma$ of the expected square of each term. Writing squares of Lebesgue integrals as double integrals, and using Itô's formula, each term's expected square is thus, up to $(m, k)$-dependent multiplicative constants, equal to the expression

$$
\begin{aligned}
K= & \frac{1}{\varepsilon^{2}} \int_{u_{m-k}=0}^{T} \int_{u_{m-k}^{\prime}=0}^{T} \int_{u_{m-k-1}=0}^{u_{m-k}} \int_{u_{m-k-1}^{\prime}=0}^{u_{m-k}} \ldots \int_{u_{1}=0}^{u_{2}} \int_{u_{1}^{\prime}=0}^{u_{2}} \mathbf{E} \\
& \times\left[\mathrm{d}[M]^{\otimes k}\left(u_{\sigma(1)}, \ldots, u_{\sigma(k)}\right) \mathrm{d}[M]^{\otimes k}\left(u_{\sigma(1)}^{\prime}, \ldots, u_{\sigma(k)}^{\prime}\right) \mathrm{d}[M]^{\otimes(m-2 k)}\left(u_{\sigma(k+1)}, \ldots, u_{\sigma(m-k)}\right)\right] \\
& \cdot\left[\Delta G \cdot\left(u_{\sigma(k+1)}\right) ; \ldots ; \Delta G \cdot\left(u_{\sigma(m-k)}\right) ; \Delta G \cdot\left(u_{\sigma(1)}\right) ; \Delta G \cdot\left(u_{\sigma(1)}\right) ; \ldots ; \Delta G \cdot\left(u_{\sigma(k)}\right) ; \Delta G \cdot\left(u_{\sigma(k)}\right)\right] \\
& \cdot\left[\Delta G \cdot\left(u_{\sigma(k+1)}\right) ; \ldots ; \Delta G \cdot\left(u_{\sigma(m-k)}\right) ; \Delta G \cdot\left(u_{\sigma(1)}^{\prime}\right) ; \Delta G \cdot\left(u_{\sigma(1)}^{\prime}\right) ; \ldots ; \Delta G \cdot\left(u_{\sigma(k)}^{\prime}\right) ; \Delta G \cdot\left(u_{\sigma(k)}^{\prime}\right)\right],
\end{aligned}
$$

modulo the fact that one may remove the integrals with respect to those $u_{j}^{\prime}$ 's not represented among $\left\{u_{\sigma(1)}^{\prime}, \ldots, u_{\sigma(k)}^{\prime}\right\}$. If we can show that for all $k \in\{0,1,2, \ldots,[m / 2]\}$ and all $\sigma \in \Sigma_{m}^{k}$, the above expression $K=K_{m, k, \sigma}$ tends to 0 as $\varepsilon$ tends to 0 , the theorem will be proved. 
A final note about notation. The bracket notation in the last two lines of the expression (A.3) above means that we have the product of two separate Riemann integrals over $s \in[0, T]$. Below we will denote these integrals as being with respect to $s \in[0, T]$ and $t \in[0, T]$.

Step 1. Diagonal. As in Step 1 of the proofs of Theorems 3.6 and 3.8, we can Cauchy-Schwarz to deal with the portion of $K_{m, k, \sigma}$ in (A.3) where $|s-t| \leq 2 \varepsilon$. The details are omitted.

Step 2. Term for $k=0$. When $k=0$, there is only one permutation $\sigma=I d$, and we have, using hypothesis (A)

$$
\begin{aligned}
K_{m, 0, I d} & =\frac{1}{\varepsilon^{2}} \int_{u_{m}=0}^{T} \int_{u_{m-1}=0}^{u_{m}} \ldots \int_{u_{1}=0}^{u_{2}} \mathbf{E}\left[\mathrm{d}[M]^{\otimes m}\left(u_{1}, \ldots, u_{m}\right)\right] \cdot\left[\Delta G \cdot\left(u_{1}\right) ; \ldots ; \Delta G \cdot\left(u_{m}\right)\right]^{2} \\
& \leq \frac{1}{\varepsilon^{2}} \int_{u_{m-k}=0}^{T} \int_{u_{m-k-1}=0}^{u_{m-k}} \ldots \int_{u_{1}=0}^{u_{2}} \Gamma^{2}\left(u_{1}\right) \Gamma^{2}\left(u_{2}\right) \ldots \Gamma^{2}\left(u_{m}\right)\left[\Delta G \cdot\left(u_{1}\right) ; \ldots ; \Delta G \cdot\left(u_{m}\right)\right]^{2} \mathrm{~d} u_{1} \mathrm{~d} u_{2} \ldots \mathrm{d} u_{m} \\
& =\frac{1}{\varepsilon^{2}} \int_{u_{m-k}=0}^{T} \int_{u_{m-k-1}=0}^{u_{m-k}} \ldots \int_{u_{1}=0}^{u_{2}}\left[\Delta \tilde{G} \cdot\left(u_{1}\right) ; \ldots ; \Delta \tilde{G} \cdot\left(u_{m}\right)\right]^{2} \mathrm{~d} u_{1} \mathrm{~d} u_{2} \ldots \mathrm{d} u_{m} .
\end{aligned}
$$

This is precisely the expression one gets for the term corresponding to $k=0$ when $M=W$, i.e. when $X$ is the Gaussian process $Z$ with kernel $\tilde{G}$. Hence our hypotheses from the previous two theorems guarantee that this expression tends to 0 .

Step 3. Term for $k=1$. Again, in this case, $\sigma=I d$, and we thus have, using hypothesis (A),

$$
\begin{aligned}
& K_{m, 1, I d}=\frac{1}{\varepsilon^{2}} \int_{u_{m-1}=0}^{T} \int_{u_{m-2}=0}^{u_{m-1}} \ldots \int_{u_{1}=0}^{u_{2}} \int_{u_{1}^{\prime}=0}^{u_{2}} \mathbf{E}\left[\mathrm{d}[M]\left(u_{1}\right) \mathrm{d}[M]\left(u_{1}^{\prime}\right) \mathrm{d}[M]^{\otimes(m-2)}\left(u_{2}, \ldots, u_{m-1}\right)\right] \\
& \cdot\left[\Delta G \cdot\left(u_{2}\right) ; \ldots ; \Delta G \cdot\left(u_{m-1}\right) ; \Delta G \cdot\left(u_{1}\right) ; \Delta G \cdot\left(u_{1}\right)\right] \cdot\left[\Delta G \cdot\left(u_{2}\right) ; \ldots ; \Delta G \cdot\left(u_{m-1}\right) ; \Delta G \cdot\left(u_{1}^{\prime}\right) ; \Delta G .\left(u_{1}^{\prime}\right)\right] \\
& \leq \frac{1}{\varepsilon^{2}} \int_{u_{m-1}=0}^{T} \int_{u_{m-2}=0}^{u_{m-1}} \ldots \int_{u_{1}=0}^{u_{2}} \int_{u_{1}^{\prime}=0}^{u_{2}} \mathrm{~d} u_{1} \mathrm{~d} u_{1}^{\prime} \mathrm{d} u_{2} \ldots \mathrm{d} u_{m-1} \Gamma^{2}\left(u_{1}\right) \Gamma^{2}\left(u_{1}^{\prime}\right) \Gamma^{2}\left(u_{2}\right) \ldots \Gamma^{2}\left(u_{m}\right) \\
& \cdot\left[|\Delta G| .\left(u_{2}\right) ; \ldots ;|\Delta G| .\left(u_{m-1}\right) ;|\Delta G| .\left(u_{1}\right) ;|\Delta G| .\left(u_{1}\right)\right] \cdot\left[|\Delta G| .\left(u_{2}\right) ; \ldots ;|\Delta G| .\left(u_{m-1}\right) ;|\Delta G| .\left(u_{1}^{\prime}\right) ;|\Delta G| .\left(u_{1}^{\prime}\right)\right] \\
& =\frac{1}{\varepsilon^{2}} \int_{u_{m-1}=0}^{T} \int_{u_{m-2}=0}^{u_{m-1}} \ldots \int_{u_{1}=0}^{u_{2}} \int_{u_{1}^{\prime}=0}^{u_{2}} \mathrm{~d} u_{1} \mathrm{~d} u_{1}^{\prime} \mathrm{d} u_{2} \ldots \mathrm{d} u_{m-1} \\
& {\left[|\Delta \tilde{G}|\left(u_{2}\right) ; \ldots ;|\Delta \tilde{G}| .\left(u_{m-1}\right) ;|\Delta \tilde{G}| .\left(u_{1}\right) ;|\Delta \tilde{G}| .\left(u_{1}\right)\right]} \\
& \cdot\left[|\Delta \tilde{G}| .\left(u_{2}\right) ; \ldots ;|\Delta \tilde{G}| .\left(u_{m-1}\right) ;|\Delta \tilde{G}| .\left(u_{1}^{\prime}\right) ;|\Delta \tilde{G}| .\left(u_{1}^{\prime}\right)\right] .
\end{aligned}
$$

Note now that the product of two bracket operators [...][..] means we integrate over $0 \leq s \leq t-2 \varepsilon$ and $2 \varepsilon \leq t \leq T$, and get an additional factor of 2 , since the diagonal term was dealt with in Step 1 .

In order to exploit the additional hypothesis (4.3) in our theorem, our first move is to use Fubini by bringing the integrals over $u_{1}$ all the way inside. We get

$$
\begin{aligned}
K_{m, 1, I d} \leq \frac{2}{\varepsilon^{2}} & \int_{u_{m-1}=0}^{T} \int_{u_{m-2}=0}^{u_{m-1}} \ldots \int_{u_{2}=0}^{u_{3}} \mathrm{~d} u_{2} \ldots \mathrm{d} u_{m-1} \\
& \int_{t=2 \varepsilon}^{T} \int_{s=0}^{t-2 \varepsilon} \mathrm{d} s \mathrm{~d} t\left|\Delta \tilde{G}_{s}\left(u_{2}\right)\right| \ldots\left|\Delta \tilde{G}_{s}\left(u_{m-1}\right)\right|\left|\Delta \tilde{G}_{t}\left(u_{2}\right)\right| \ldots\left|\Delta \tilde{G}_{t}\left(u_{m-1}\right)\right| \\
& \int_{u_{1}=0}^{u_{2}} \int_{u_{1}^{\prime}=0}^{u_{2}} \mathrm{~d} u_{1} \mathrm{~d} u_{1}^{\prime}\left(\Delta \tilde{G}_{s}\left(u_{1}\right)\right)^{2}\left(\Delta \tilde{G}_{t}\left(u_{1}^{\prime}\right)\right)^{2} .
\end{aligned}
$$


The term in the last line above is trivially bounded above by

$$
\int_{u_{1}=0}^{T} \int_{u_{1}^{\prime}=0}^{T} \mathrm{~d} u_{1} \mathrm{~d} u_{1}^{\prime}\left(\Delta \tilde{G}_{s}\left(u_{1}\right)\right)^{2}\left(\Delta \tilde{G}_{t}\left(u_{1}^{\prime}\right)\right)^{2}
$$

precisely equal to $\operatorname{Var}[Z(s+\varepsilon)-Z(s)] \operatorname{Var}[Z(t+\varepsilon)-Z(t)]$, which by hypothesis is bounded above by $\delta^{4}(\varepsilon)$. Consequently, we get

$$
\begin{aligned}
K_{m, 1, I d} & \leq 2 \frac{\delta^{4}(\varepsilon)}{\varepsilon^{2}} \int_{u_{m-1}=0}^{T} \int_{u_{m-2}=0}^{u_{m-1}} \ldots \int_{u_{2}=0}^{u_{3}} \mathrm{~d} u_{2} \ldots \mathrm{d} u_{m-1} \\
& \int_{t=2 \varepsilon}^{T} \int_{s=0}^{t-2 \varepsilon} \mathrm{d} s \mathrm{~d} t\left|\Delta \tilde{G}_{s}\left(u_{2}\right)\right| \ldots\left|\Delta \tilde{G}_{s}\left(u_{m-1}\right)\right|\left|\Delta \tilde{G}_{t}\left(u_{2}\right)\right| \ldots\left|\Delta \tilde{G}_{t}\left(u_{m-1}\right)\right| .
\end{aligned}
$$

We get an upper bound by integrating all the $u_{j}$ 's over their entire range $[0, T]$, i.e. we have,

$$
\begin{aligned}
& K_{m, 1, I d} \leq \frac{\delta^{4}(\varepsilon)}{\varepsilon^{2}} \int_{t=2 \varepsilon}^{T} \mathrm{~d} t \int_{s=0}^{t-2 \varepsilon} \mathrm{d} s \\
& \int_{0}^{T} \int_{0}^{T} \ldots \int_{0}^{T} \mathrm{~d} u_{3} \ldots \mathrm{d} u_{m-1}\left|\Delta \tilde{G}_{s}\left(u_{3}\right)\right| \ldots\left|\Delta \tilde{G}_{s}\left(u_{m-1}\right)\right|\left|\Delta \tilde{G}_{t}\left(u_{3}\right)\right| \ldots\left|\Delta \tilde{G}_{t}\left(u_{m-1}\right)\right| \\
& \cdot \int_{u_{2}=0}^{T}\left|\Delta \tilde{G}_{t}\left(u_{2}\right)\right|\left|\Delta \tilde{G}_{s}\left(u_{2}\right)\right| \mathrm{d} u_{2} \\
& =2 \frac{\delta^{4}(\varepsilon)}{\varepsilon^{2}} \int_{t=2 \varepsilon}^{T} \mathrm{~d} t \int_{s=0}^{t-2 \varepsilon} \mathrm{d} s\left(\int_{0}^{T} \mathrm{~d} u\left|\Delta \tilde{G}_{s}(u)\right|\left|\Delta \tilde{G}_{t}(u)\right|\right)^{m-3} \cdot \int_{u_{2}=0}^{u_{3}}\left|\Delta \tilde{G}_{t}\left(u_{2}\right)\right|\left|\Delta \tilde{G}_{s}\left(u_{2}\right)\right| \mathrm{d} u_{2} .
\end{aligned}
$$

Now we use a simple Cauchy-Schwarz's inequality for the integral over $u$, but not for $u_{2}$. Recognizing that $\int_{0}^{T}\left|\Delta \tilde{G}_{s}(u)\right|^{2} \mathrm{~d} u$ is the variance $\operatorname{Var}[Z(s+\varepsilon)-Z(s)] \leq \delta^{2}(\varepsilon)$, we have

$$
\begin{aligned}
K_{m, 1, I d} & \leq 2 \frac{\delta^{4}(\varepsilon)}{\varepsilon^{2}} \int_{t=2 \varepsilon}^{T} \mathrm{~d} t \int_{s=0}^{t-2 \varepsilon} \mathrm{d} s\left(\int_{0}^{T} \mathrm{~d} u\left|\Delta \tilde{G}_{s}(u)\right|^{2}\right)^{m-3} \cdot \int_{u_{2}=0}^{u_{3}}\left|\Delta \tilde{G}_{t}\left(u_{2}\right)\right|\left|\Delta \tilde{G}_{s}\left(u_{2}\right)\right| \mathrm{d} u_{2} . \\
& \leq 2 \frac{\delta^{4+2 m-6}(\varepsilon)}{\varepsilon^{2}} \int_{t=2 \varepsilon}^{T} \mathrm{~d} t \int_{s=0}^{t-2 \varepsilon} \mathrm{d} s \int_{u_{2}=0}^{T}\left|\Delta \tilde{G}_{t}\left(u_{2}\right)\right|\left|\Delta \tilde{G}_{s}\left(u_{2}\right)\right| \mathrm{d} u_{2} .
\end{aligned}
$$

Condition (4.3) implies immediately $K_{m, 1, I d} \leq \delta^{2 m}(2 \varepsilon) \varepsilon^{-1}$ which tends to 0 with $\varepsilon$ by hypothesis.

Step 4. $k \geq 2$. This step proceeds using the same technique as Step 3. Fix $k \geq 2$. Now for each given permutation $\sigma$, there are $k$ pairs of parameters of the type $\left(u, u^{\prime}\right)$. Each of these contributes precisely a term $\delta^{4}(\varepsilon)$, as in the previous step, i.e. $\delta^{4 k}(\varepsilon)$ altogether. In other words, for every $\sigma \in \Sigma_{m}^{k}$, and deleting the diagonal term, we have

$$
\begin{aligned}
& K_{m, k, \sigma} \\
& \leq 2 \frac{\delta^{4 k}(\varepsilon)}{\varepsilon^{2}} \int_{t=2 \varepsilon}^{T} \mathrm{~d} t \int_{s=0}^{t-2 \varepsilon} \mathrm{d} s \int_{0}^{T} \int_{0}^{u_{m-k}} \ldots \int_{0}^{u_{k+2}} \mathrm{~d} u_{k+1} \ldots \mathrm{d} u_{m-k}\left[\int_{0}^{T} \mathrm{~d} s\left|\Delta \tilde{G}_{s}\left(u_{k+1}\right)\right| \ldots\left|\Delta \tilde{G}_{s}\left(u_{m-k}\right)\right|\right]^{2} .
\end{aligned}
$$

Since $k \leq(m-1) / 2$, there is at least one integral, the one in $u_{k+1}$, above. We treat all the remaining integrals, if any, over $u_{k+2}, \ldots, u_{m-k}$ with Cauchy-Schwarz's inequality as in Step 3, yielding a contribution $\delta^{2(m-2 k-1)}(\varepsilon)$. The remaining integral over $u_{k+1}$ yields, by condition (4.3), a contribution of $\delta^{2}(2 \varepsilon) \varepsilon$. Hence the contribution of $K_{m, k, \sigma}$ is again $\delta^{2 m}(2 \varepsilon) \varepsilon^{-1}$, which tends to 0 with $\varepsilon$ by hypothesis, concluding the proof of the Theorem. 
Proof of Proposition 4.2. Below the value $1 /(2 m)-1 / 2$ is denoted by $\alpha$. We now show that we can apply Theorem 3.8 directly to the Gaussian process $Z$ given in (4.2), which, by Theorem 4.1, is sufficient, together with Condition (4.3), to obtain our desired conclusion. Note the assumption about $\tilde{G}$ implies that $s \mapsto \tilde{G}(t, s)$ is square-integrable, and therefore $Z$ is well-defined. We will prove condition (3.6) holds in Step 1; Step 2 will show condition (3.7) holds; Condition (4.3) will be established in Step 3.

Step 1. Variance calculation. We need only to show $\tilde{\delta}^{2}(s, s+\varepsilon)=o\left(\varepsilon^{1 / m}\right)$ uniformly in $s$. We have, for given $s$ and $t=s+\varepsilon$

$$
\begin{aligned}
\tilde{\delta}^{2}(s, s+\varepsilon)= & \int_{0}^{s}\left|(s+\varepsilon-r)^{\alpha} \tilde{f}(s+\varepsilon, r)-(s-r)^{\alpha} \tilde{f}(s, r)\right|^{2} \mathrm{~d} r \\
& +\int_{s}^{s+\varepsilon}|s+\varepsilon-r|^{2 \alpha} \tilde{f}^{2}(s+\varepsilon, r) \mathrm{d} r=: A+B .
\end{aligned}
$$

Since $\tilde{f}^{2}(s+\varepsilon, r) \leq f(s+\varepsilon-r)$ and the univariate $f$ increases, in $B$ we can bound this last quantity by $f(\varepsilon)$, and we get

$$
B \leq f^{2}(\varepsilon) \int_{0}^{\varepsilon} r^{2 \alpha} \mathrm{d} r=3 f^{2}(\varepsilon) \varepsilon^{2 \alpha+1}=o\left(\varepsilon^{1 / m}\right)
$$

The term $A$ is slightly more delicate to estimate. Since $\tilde{f}$ is increasing and $g$ is decreasing in $t$,

$$
\begin{aligned}
A & \leq \int_{0}^{s} \tilde{f}^{2}(s+\varepsilon, r)\left|(s+\varepsilon-r)^{\alpha}-(s-r)^{\alpha}\right|^{2} \mathrm{~d} r=\int_{0}^{s} f^{2}(\varepsilon+r)\left|r^{\alpha}-(r+\varepsilon)^{\alpha}\right|^{2} \mathrm{~d} r \\
& =\int_{0}^{\varepsilon} f^{2}(\varepsilon+r)\left|r^{\alpha}-(r+\varepsilon)^{\alpha}\right|^{2} \mathrm{~d} r+\int_{\varepsilon}^{s} f^{2}(\varepsilon+r)\left|r^{\alpha}-(r+\varepsilon)^{\alpha}\right|^{2} \mathrm{~d} r \\
& =: A_{1}+A_{2} .
\end{aligned}
$$

We have, again from the univariate $f$ 's increasingness, and the $\operatorname{limit}_{\lim } \rightarrow 0 f(r)=0$,

$$
A_{1} \leq f^{2}(2 \varepsilon) \int_{0}^{\varepsilon}\left|r^{\alpha}-(r+\varepsilon)^{\alpha}\right|^{2} \mathrm{~d} r=\mathrm{cst} \cdot f^{2}(2 \varepsilon) \varepsilon^{2 \alpha+1}=o\left(\varepsilon^{1 / m}\right)
$$

For the other part of $A$, we need to use f's concavity at the point $2 \varepsilon$ in the interval $[0, \varepsilon+r]$ (since $\varepsilon+r>2 \varepsilon$ in this case), which implies $f(\varepsilon+r)<f(2 \varepsilon)(\varepsilon+r) /(2 \varepsilon)$. Also using the mean-value theorem for the difference of negative cubes, we get

$$
\begin{aligned}
A_{2} & \leq \mathrm{cst} \cdot \varepsilon^{2} \int_{\varepsilon}^{s} f^{2}(\varepsilon+r) r^{2 \alpha-2} \mathrm{~d} r \leq \mathrm{cst} \cdot \varepsilon f(2 \varepsilon) \int_{\varepsilon}^{s}(\varepsilon+r) r^{2 \alpha-2} \mathrm{~d} r \\
& \leq \mathrm{cst} \cdot \varepsilon f(2 \varepsilon) \int_{\varepsilon}^{s} r^{2 \alpha-1}=\mathrm{cst} \cdot \varepsilon^{2 \alpha+1} f(2 \varepsilon)=o\left(\varepsilon^{1 / 3}\right) .
\end{aligned}
$$

This finishes the proof of Condition (3.6).

Step 2. Covariance calculation. We first calculate the second mixed derivative $\partial^{2} \tilde{\delta}^{2} / \partial s \partial t$, where $\tilde{\delta}$ is the canonical metric of $Z$, because we must show $|\mu|(O D) \leq \varepsilon^{2 \alpha}$, which is condition (3.7), and $\mu(\mathrm{d} s \mathrm{~d} t)=\mathrm{d} s \mathrm{~d} t \partial^{2} \tilde{\delta}^{2} / \partial s \partial t$. We have, for $0 \leq s \leq t-\varepsilon$,

$$
\tilde{\delta}^{2}(s, t)=\int_{0}^{s}(g(t, s-r)-g(s, s-r))^{2} \mathrm{~d} r+\int_{s}^{t} g^{2}(t, r) \mathrm{d} r=: A+B .
$$


We calculate

$$
\begin{aligned}
\frac{\partial^{2} A}{\partial s \partial t}(t, s)= & 2 \frac{\partial g}{\partial t}(t, 0)(g(t, 0)-g(s, 0)) \\
& +\int_{0}^{s} 2 \frac{\partial g}{\partial t}(t, s-r)\left(\frac{\partial g}{\partial s}(t, s-r)-\frac{\partial g}{\partial t}(s, s-r)-\frac{\partial g}{\partial s}(s, s-r)\right) \\
& +\int_{0}^{s} 2(g(t, s-r)-g(s, s-r)) \frac{\partial^{2} g}{\partial s \partial t}(t, s-r) \mathrm{d} r . \\
= & A_{1}+A_{2}+A_{3},
\end{aligned}
$$

and

$$
\frac{\partial^{2} B}{\partial s \partial t}(t, s)=-2 g(t, s) \frac{\partial g}{\partial t}(t, s)
$$

Next, we immediately get, for the portion of $|\mu|(O D)$ corresponding to $B$, using the hypotheses of our proposition,

$$
\begin{aligned}
\int_{\varepsilon}^{T} \mathrm{~d} t \int_{0}^{t-\varepsilon} \mathrm{d} s\left|\frac{\partial^{2} B}{\partial s \partial t}(t, s)\right| & \leq 2 c \int_{\varepsilon}^{T} \mathrm{~d} t \int_{0}^{t-\varepsilon} \mathrm{d} s f(|t-s|)|t-s|^{\alpha}|t-s|^{\alpha-1} \\
& \leq 2 c\|f\|_{\infty} \int_{\varepsilon}^{T} \mathrm{~d} t \varepsilon^{2 \alpha}=\mathrm{cst} \cdot \varepsilon^{2 \alpha}
\end{aligned}
$$

which is of the correct order for condition (3.7). For the term corresponding to $A_{1}$, using our hypotheses, we have

$$
\int_{\varepsilon}^{T} \mathrm{~d} t \int_{0}^{t-\varepsilon} \mathrm{d} s\left|A_{1}\right| \leq 2 \int_{\varepsilon}^{T} \mathrm{~d} t \int_{0}^{t-\varepsilon} \mathrm{d} s t^{\alpha}\left|\frac{\partial g}{\partial t}\left(\xi_{t, s}, 0\right)\right||t-s|
$$

where $\xi_{t, s}$ is in the interval $(s, t)$. Our hypothesis thus implies $\left|\frac{\partial g}{\partial t}\left(\xi_{t, s}, 0\right)\right| \leq s^{\alpha}$, and hence

$$
\int_{\varepsilon}^{T} \mathrm{~d} t \int_{0}^{t-\varepsilon} \mathrm{d} s\left|A_{1}\right| \leq 2 T \int_{\varepsilon}^{T} \mathrm{~d} t \int_{0}^{t-\varepsilon} \mathrm{d} s s^{\alpha-1} t^{\alpha-1}=2 T \alpha^{-1} \int_{\varepsilon}^{T} \mathrm{~d} t t^{\alpha-1}(t-\varepsilon)^{\alpha} \leq \alpha^{-2} T^{1+2 \alpha} .
$$

This is much smaller than the right-hand side $\varepsilon^{2 \alpha}$ of condition (3.7), since $2 \alpha=1 / m-1<0$. The terms $A_{2}$ and $A_{3}$ are treated similarly, thanks to our hypotheses.

Step 3. Proving condition (4.3). We modify the proof of Theorem 4.1, in particular Steps 3 and 4, so that we only need to prove

$$
\int_{t=2 \varepsilon}^{T} \mathrm{~d} t \int_{s=0}^{t-2 \varepsilon} \mathrm{d} s \int_{u=0}^{T}\left|\Delta \tilde{G}_{t}(u)\right|\left|\Delta \tilde{G}_{s}(u)\right| \mathrm{d} u \leq c \varepsilon^{2+2 \alpha}=c \varepsilon^{1 / m+1},
$$

instead of condition (4.3). Indeed, for instance in Step 3, this new condition yields a final contribution of order $\delta^{2 m-2}(\varepsilon) \varepsilon^{-2} \varepsilon^{1 / m+1}$. With the assumption on $\delta$ that we have, $\delta(\varepsilon)=o\left(\varepsilon^{1 /(2 m)}\right)$, and hence the final contribution is of order $o\left(\varepsilon^{(2 m-2) /(2 m)-1+1 / m}\right)=o(1)$. This proves that the conclusion of Theorem 4.1 holds if we assume (A.5) instead of condition (4.3).

We now prove (A.5). We can write

$$
\begin{aligned}
& \int_{t=2 \varepsilon}^{T} \mathrm{~d} t \int_{s=0}^{t-2 \varepsilon} \mathrm{d} s \int_{u=0}^{T}\left|\Delta \tilde{G}_{t}(u)\right|\left|\Delta \tilde{G}_{s}(u)\right| \mathrm{d} u \\
= & \int_{t=2 \varepsilon}^{T} \mathrm{~d} t \int_{s=0}^{t-2 \varepsilon} \mathrm{d} s \int_{0}^{s}|g(t+\varepsilon, u)-g(t, u)||g(s+\varepsilon, u)-g(s, u)| \mathrm{d} u \\
& +\int_{t=2 \varepsilon}^{T} \mathrm{~d} t \int_{s=0}^{t-2 \varepsilon} \mathrm{d} s \int_{s}^{s+\varepsilon}|g(t+\varepsilon, u)-g(t, u)||g(s+\varepsilon, u)| \mathrm{d} u=: A+B .
\end{aligned}
$$


For $A$, we use the hypotheses of this proposition: for the last factor in $A$, we exploit the fact that $g$ is decreasing in $t$ while $\tilde{f}$ is increasing in $t$; for the other factor in $A$, use the bound on $\partial g / \partial t$; thus we have

$$
A \leq \int_{t=2 \varepsilon}^{T} \mathrm{~d} t \int_{s=0}^{t-2 \varepsilon} \varepsilon|t-s|^{\alpha-1} \mathrm{~d} s \int_{0}^{s} \tilde{f}(s+\varepsilon, u)\left((s-u)^{\alpha}-(s+\varepsilon-u)^{\alpha}\right) \mathrm{d} u .
$$

We separate the integral in $u$ into two pieces, for $u \in[0, s-\varepsilon]$ and $u \in[s-\varepsilon, s]$. For the first integral in $u$, since $\tilde{f}$ is bounded, we have

$$
\int_{0}^{s-\varepsilon} \tilde{f}(s+\varepsilon, u)\left((s-u)^{\alpha}-(s+\varepsilon-u)^{\alpha}\right) \mathrm{d} u \leq\|\tilde{f}\|_{\infty} \varepsilon \int_{0}^{s-\varepsilon}(s-u)^{\alpha-1} \mathrm{~d} u \leq\|\tilde{f}\|_{\infty} c_{\alpha} \varepsilon^{1+\alpha} .
$$

For the second integral in $u$, we use the fact that $s-u+\varepsilon>\varepsilon$ and $s-u<\varepsilon$ implies $s-u+\varepsilon>2(s-u)$, so that the negative part of the integral can be ignored, and thus

$$
\int_{s-\varepsilon}^{s} \tilde{f}(s+\varepsilon, u)\left((s-u)^{\alpha}-(s+\varepsilon-u)^{\alpha}\right) \mathrm{d} u \leq\|\tilde{f}\|_{\infty} \int_{s-\varepsilon}^{s}(s-u)^{\alpha} \mathrm{d} u=\|\tilde{f}\|_{\infty} c_{\alpha} \varepsilon^{1+\alpha},
$$

which is the same upper bound as for the other part of the integral in $u$. Thus

$$
A \leq \operatorname{cst} \cdot \varepsilon^{2+\alpha} \int_{t=2 \varepsilon}^{T} \mathrm{~d} t \int_{s=0}^{t-2 \varepsilon}|t-s|^{\alpha-1} \mathrm{~d} s \leq \operatorname{cst} \cdot \varepsilon^{2+\alpha} \int_{t=2 \varepsilon}^{T} \mathrm{~d} t \varepsilon^{\alpha} \leq \operatorname{cst} \cdot \varepsilon^{2+2 \alpha}=\operatorname{cst} \cdot \varepsilon^{1 / m+1},
$$

which is the conclusion we needed at least for $A$.

Lastly, we estimate $B$. We use the fact that $\tilde{f}$ is bounded, and thus $|g(s+\varepsilon, u)| \leq\|\tilde{f}\|_{\infty}|s+\varepsilon-u|^{\alpha}$, as well as the estimate on the derivative of $g$ as we did in the calculation of $A$, yielding

$$
\begin{aligned}
B & \leq\|\tilde{f}\|_{\infty} \varepsilon \int_{t=2 \varepsilon}^{T} \mathrm{~d} t \int_{s=0}^{t-2 \varepsilon} \mathrm{d} s|t-s-\varepsilon|^{\alpha-1} \int_{s}^{s+\varepsilon}|s+\varepsilon-u|^{\alpha} \mathrm{d} u \\
& =\operatorname{cst} \cdot \varepsilon^{\alpha+2} \int_{t=2 \varepsilon}^{T} \mathrm{~d} t \int_{s=0}^{t-2 \varepsilon} \mathrm{d} s|t-s-\varepsilon|^{\alpha-1} \\
& \leq 2^{1+|\alpha|} \mathrm{cst} \cdot \varepsilon^{\alpha+2} \int_{t=2 \varepsilon}^{T} \mathrm{~d} t \int_{s=0}^{t-2 \varepsilon} \mathrm{d} s|t-s|^{\alpha-1} \leq \mathrm{cst} \cdot \varepsilon^{2 \alpha+2}=\mathrm{cst} \cdot \varepsilon^{1 / m+1} .
\end{aligned}
$$

This is the conclusion we needed for $B$, which finishes the proof of the proposition.

\section{REFERENCES}

[1] R. Adler, An introduction to continuity, extrema, and related topics for general Gaussian processes. Inst. Math. Stat. Hayward, CA (1990).

[2] E. Alòs and D. Nualart, Stochastic integration with respect to fractional Brownian motion. Stoch. Stoch. Rep. 75 (2003) 129-152.

[3] E. Alòs, O. Mazet and D. Nualart, Stochastic calculus with respect to Gaussian processes. Ann. Probab. 29 (1999) $766-801$.

[4] J.-M. Azaïs and M. Wschebor, Almost sure oscillation of certain random processes. Bernoulli 2 (1996) $257-270$.

[5] J. Bertoin, Sur une intégrale pour les processus à $\alpha$-variation bornée. Ann. Probab. 17 (1989) 1521-1535.

[6] F. Biagini, Y. Hu, B. Øksendal and T. Zhang, Stochastic calculus with respect to fractional Brownian motion and applications. Probab. Appl. Springer-Verlag (2008).

[7] C. Borell, On polynomial chaos and integrability. Probab. Math. Statist. 3 (1984) 191-203.

[8] P. Breuer and P. Major, Central limit theorems for non-linear functionals of Gaussian fields. J. Multivariate Anal. 13 (1983) 425-441.

[9] M. Bruneau, Variation totale d'une fonction. Vol. 413 of Lect. Notes Math. Springer-Verlag, Berlin-New York (1974).

[10] K. Burdzy, and J. Swanson, A change of variable formula with Itô correction term. Ann. Probab. 38 (2010) $1817-1869$.

[11] Ph, Carmona, L. Coutin and G. Montseny, Stochastic integration with respect to fractional Brownian motion. Ann. Inst. Henri Poincaré, Probab. Statist. 39 (2003) 27-68. 
[12] R.M. Dudley and R. Norvaiša, Differentiability of six operators on nonsmooth functions and $p$-variation. With the collaboration of Jinghua Qian. Vol. 1703 of Lect. Notes Math. Springer-Verlag, Berlin (1999).

[13] M. Errami and F. Russo, Covariation de convolution de martingales. C. R. Acad. Sci. Paris Sér. I Math. 326 (1998) $601-606$.

[14] M. Errami and F. Russo, $n$-covariation,generalized Dirichlet processes and calculus with respect to finite cubic variation processes. Stoch. Process. Appl. 104 (2003) 259-299.

[15] F. Flandoli and F. Russo, Generalized stochastic integration and stochastic ODE's. Ann. Probab. 30 (2002) $270-292$.

[16] H. Föllmer, Calcul d'Itô sans probabilités. In Séminaire de Probabilités, XV, Univ. Strasbourg, Strasbourg, $1979 / 1980$ (French). In vol. 850 of Lect. Notes Math. Springer, Berlin (1981) 143-150.

[17] P. Friz and N. Victoir, Multidimensional Stochastic Processes as Rough Paths: Theory and Applications. Cambridge Stud. Adv. Math. Cambridge UP (2010).

[18] M. Gradinaru and I. Nourdin, Approximation at first and second order of $m$-order integrals of the fractional Brownian motion and of certain semimartingales. Electron. J. Probab. 8 (2003) 26.

[19] M. Gradinaru, F. Russo and P. Vallois, Generalized covariations, local time and Stratonovich Itô's formula for fractional Brownian motion with Hurst index $H \geq \frac{1}{4}$. Ann. Pobab. 31 (2003) 1772-1820.

[20] M. Gradinaru, I. Nourdin, F. Russo and P. Vallois, $m$-order integrals and Itô's formula for non-semimartingale processes; the case of a fractional Brownian motion with any Hurst index. Ann. Inst. Henri Poincaré, Probab. Stat. 41 (2005) 781-806.

[21] Y. Hu, D. Nualart and J. Song, Fractional martingales and characterization of the fractional Brownian motion. Ann. Probab. 37 (2009) 2404-2430.

[22] I. Kruk and F. Russo, Malliavin-Skorohod calculus and Paley-Wiener integral for covariance singular processes. Preprint HAL-INRIA 00540914.

[23] H.-H. Kuo, Introduction to stochastic integration. Springer (2006).

[24] T. Lyons and Z. Qian, System control and rough paths. Oxford Math. Monogr. Oxford University Press, Oxford (2002).

[25] O. Mocioalca and F. Viens, Skorohod integration and stochastic calculus beyond the fractional Brownian scale. J. Funct. Anal. 222 (2004) 385-434.

[26] I. Nourdin, A change of variable formula for the 2D fractional Brownian motion of Hurst index bigger or equal to 1/4. J. Funct. Anal. 256 (2009) 2304-2320.

[27] I. Nourdin and D. Nualart, Central limit theorems for multiple Skorohod integrals. J. Theoret. Probab. 23 39-64.

[28] I. Nourdin, D. Nualart and C. Tudor, Central and non-central limit theorems for weighted power variations of fractional Brownian motion. Ann. Inst. Henri Poincaré, Probab. Stat. 46 (2010) 1055-1079.

[29] I. Nourdin, A. Réveillac and J. Swanson, The weak Stratonovich integral with respect to fractional Brownian motion with Hurst parameter 1/6. Electron. J. Probab. 15 (2010) 2117-2162.

[30] D. Nualart, The Malliavin calculus and related topics, 2nd edition. Probab. Appl. Springer-Verlag (2006).

[31] D. Nualart and S. Ortiz-Latorre, Central limit theorems for multiple stochastic integrals and Malliavin calculus. Stochastic Process. Appl. 118 (2008) 614-628.

[32] L.C.G. Rogers and J.B. Walsh, The exact 4/3-variation of a process arising from Brownian motion. Stoch. Stoch. Rep. 51 (1994) 267-291.

[33] F. Russo and C. Tudor, On the bifractional Brownian motion. Stoch. Process. Appl. 116 (2006) 830-856.

[34] F. Russo and P. Vallois, The generalized covariation process and Itô formula. Stoch. Process. Appl. 59 (1995) 81-104.

[35] F. Russo and P. Vallois, Stochastic calculus with respect to a finite quadratic variation process. Stoch. Stoch. Rep. 70 (2000) $1-40$.

[36] F. Russo and P. Vallois, Elements of stochastic calculus via regularizations. Séminaire de Probabilités XL. Vol. 1899 of Lect. Notes Math. Springer, Berlin Heidelberg, New-York (2007) 147-186.

[37] F. Russo and F. Viens, Gaussian and non-Gaussian processes of zero power variation, and related stochastic calculus. Preprint arXiv: 1407.4568 (2014).

[38] S. Tindel, C.A. Tudor and F. Viens, Sharp Gaussian regularity on the circle and application to the fractional stochastic heat equation. J. Funct. Anal. 217 (2004) 280-313.

[39] F. Viens and A. Vizcarra, Supremum Concentration Inequality and Modulus of Continuity for Sub- $n$th Chaos Processes. J. Funct. Anal. 248 (2007) 1-26. 